\title{
ZTF Early Observations of Type Ia Supernovae. I. Properties of the 2018 Sample
}

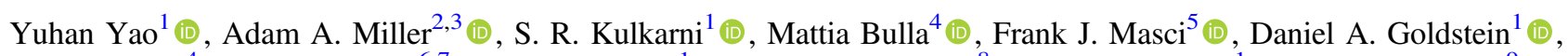

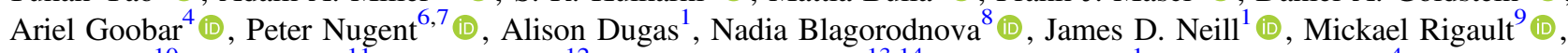
Jesper Sollerman ${ }^{10}$ (1) , J. Nordin ${ }^{11}$, Eric C. Bellm ${ }^{12}$ (1) , S. Bradley Cenko ${ }^{13,14}$ (1) , Kishalay De ${ }^{1}$ (i), Suhail Dhawan ${ }^{4}$, Ulrich Feindt ${ }^{4}$ (1),

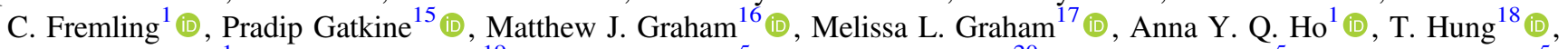

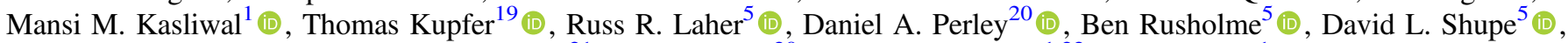
Maayane T. Soumagnac ${ }^{21}$ (1) K. Taggart ${ }^{20}$ (1) , Richard Walters ${ }^{1,22}$, and Lin Yan $^{1}$ (1)

${ }_{1}^{1}$ Cahill Center for Astrophysics, California Institute of Technology, MC 249-17, 1200 E California Boulevard, Pasadena, CA 91125, USA; yyao@astro.caltech.edu

2 Center for Interdisciplinary Exploration and Research in Astrophysics (CIERA) and Department of Physics and Astronomy, Northwestern University, 2145 Sheridan Road, Evanston, IL 60208, USA

${ }^{3}$ The Adler Planetarium, Chicago, IL 60605, USA

${ }^{4}$ The Oskar Klein Centre, Department of Physics, Stockholm University, AlbaNova, SE-106 91 Stockholm, Sweden

${ }^{5}$ IPAC, California Institute of Technology, 1200 E. California Blvd, Pasadena, CA 91125, USA

${ }^{6}$ Computational Cosmology Center, Lawrence Berkeley National Laboratory, 1 Cyclotron Road, Berkeley, CA 94720, USA

${ }_{8}^{7}$ Department of Astronomy, University of California, Berkeley, CA 94720-3411, USA

${ }^{8}$ Department of Astrophysics/IMAPP, Radboud University, Nijmegen, The Netherlands

${ }^{9}$ Université Clermont Auvergne, CNRS/IN2P3, Laboratoire de Physique de Clermont, F-63000 Clermont-Ferrand, France

${ }^{10}$ The Oskar Klein Centre, Department of Astronomy, Stockholm University, AlbaNova, SE-10691 Stockholm, Sweden

${ }^{11}$ Institute of Physics, Humboldt-Universität zu Berlin, Newtonstr. 15, D-12489 Berlin, Germany

${ }_{12}$ DIRAC Institute, Department of Astronomy, University of Washington, 3910 15th Avenue NE, Seattle, WA 98195, USA

${ }^{13}$ Astrophysics Science Division, NASA Goddard Space Flight Center, 8800 Greenbelt Road, Greenbelt, MD 20771, USA

${ }_{15}^{14}$ Joint Space-Science Institute, University of Maryland, College Park, MD 20742, USA

${ }^{15}$ Department of Astronomy, University of Maryland, College Park, MD 20742, USA

${ }^{16}$ Division of Physics, Mathematics, and Astronomy, California Institute of Technology, Pasadena, CA 91125, USA

${ }_{17}^{17}$ University of Washington, Department of Astronomy, Box 351580, Seattle, WA 98195-1580, USA

${ }^{18}$ Department of Astronomy and Astrophysics, University of California, Santa Cruz, CA 95064, USA

${ }^{19}$ Kavli Institute for Theoretical Physics, University of California Santa-Barbara, Santa Barbara, CA 93106, USA

${ }^{20}$ Astrophysics Research Institute, Liverpool John Moores University, IC2, Liverpool Science Park, 146 Brownlow Hill, Liverpool L3 5RF, UK

${ }^{21}$ Benoziyo Center for Astrophysics, Weizmann Institute of Science, Rehovot, Israel

${ }^{22}$ Caltech Optical Observatories, California Institute of Technology, MC 249-17, 1200 E California Boulevard, Pasadena, CA 91125, USA

Received 2019 July 22; revised 2019 September 27; accepted 2019 October 5; published 2019 December 3

\begin{abstract}
Early-time observations of Type Ia supernovae (SNe Ia) are essential to constrain the properties of their progenitors. In this paper, we present high-quality light curves of 127 SNe Ia discovered by the Zwicky Transient Facility (ZTF) in 2018. We describe our method to perform forced point-spread function photometry, which can be applied to other types of extragalactic transients. With a planned cadence of six observations per night (three $g+$ three $r$ ), all of the 127 SNe Ia are detected in both $g$ and $r$ bands more than 10 days (in the rest frame) prior to the epoch of $g$-band maximum light. The redshifts of these objects range from $z=0.0181$ to 0.165 ; the median redshift is 0.074 . Among the $127 \mathrm{SNe}$, 50 are detected at least 14 days prior to maximum light (in the rest frame), with a subset of nine objects being detected more than 17 days before $g$-band peak. This is the largest sample of young SNe Ia collected to date; it can be used to study the shape and color evolution of the rising light curves in unprecedented detail. We discuss six peculiar events in this sample: one 02cx-like event ZTF18abclfee (SN 2018crl), one Ia-CSM SN ZTF18aaykjei (SN 2018cxk), and four objects with possible super-Chandrasekhar mass progenitors: ZTF18abhpgje (SN 2018eul), ZTF18abdpvnd (SN 2018dvf), ZTF18aawpcel (SN 2018cir), and ZTF18abddmrf (SN 2018dsx).
\end{abstract}

Unified Astronomy Thesaurus concepts: Type Ia supernovae (1728); Sky surveys (1464); Catalogs (205); Supernovae (1668); Surveys (1671); Photometry (1234)

Supporting material: machine-readable tables, FITS file

\section{Introduction}

Despite being used as standardizable candles to study cosmology, the origin of Type Ia supernovae (SNe Ia) is not settled (see review by Maoz et al. 2014). Two major formation channels have been proposed: single degenerate (SD), where a carbon/oxygen white dwarf (WD) accretes matter from a nondegenerate star and triggers an explosion near the Chandrasekhar mass $\left(M_{\text {ch }}\right.$, Whelan \& Iben 1973), and double degenerate (DD), in which the primary WD accretes material from (or merges with) another WD (Woosley \& Weaver 1994; Tutukov \& Yungelson 1996; Shen 2015).
Observations obtained in the hours to days after explosion (i.e., "early-time") provide a path toward diagnosing the various explosion mechanisms (Maoz et al. 2014). Early photometry can constrain the radii of the possible companion and the progenitor star (Kasen 2010; Nugent et al. 2011; Bloom et al. 2012; Goobar et al. 2014, 2015). The shape and duration of the rising light curves probe the radial distribution of radioactive ${ }^{56} \mathrm{Ni}$ in the exploding core, as well as the existence of circumstellar material (Dessart et al. 2014; Piro \& Nakar 2014; Firth et al. 2015; Piro \& Morozova 2016; Miller et al. 2018). 
Simulations of the double detonation of a helium shell on the surface of a WD predict an unusually red excess well before peak luminosity (Noebauer et al. 2017; Maeda et al. 2018; Polin et al. 2019), which was observed in SN 2016jhr (Jiang et al. 2017) and SN 2018byg (De et al. 2019). Should the SD channel hold, a collision between the SN ejecta and the stellar companion will give rise to strong ultraviolet (UV) emission at early times (Hayden et al. 2010a; Kasen 2010). The detection of a declining UV pulse in the peculiar SN Ia iPTF 14atg reasonably favors this scenario (Cao et al. 2015), although Kromer et al. (2016) argue that its spectral evolution is more consistent with a merger product. The power of well-sampled early-time photometry has also been demonstrated in single-object studies of normal SNe Ia (Marion et al. 2016; Hosseinzadeh et al. 2017; Dimitriadis et al. 2019; Shappee et al. 2019, Li et al. 2019), where the clearly resolved early bumps in their light curves pose challenges to simple explosion models.

Up to now there has not been a large ( $>100$ objects) uniform data set of SN Ia light curves with both multi-band photometry and dense early-time sampling. With the Zwicky Transient Facility (ZTF, Bellm et al. 2019b; Graham et al. 2019), we are undertaking a high-cadence survey with six epochs per night (three $g+$ three $r$; Bellm et al. 2019a). This experiment is conducted over a large area of the sky $\left(\sim 2500 \mathrm{deg}^{2}\right)$ and thus enables large-number statistics. In this study, we focus on a special subset of SNe Ia that were discovered more than 10 days prior to maximum light. Our large (127 objects), homogeneous sample of young SNe Ia was constructed within the first year of operations by ZTF.

As the first in a series of three papers, we present the light curves and sample properties of $127 \mathrm{SNe}$ Ia. A detailed analysis of the early evolution of these $\mathrm{SNe}$ will be addressed will be addressed in A.A. Miller et al. (2019, in preparation) and M. Bulla et al. (2019, in preparation). Throughout this paper, we assume a flat $\Lambda \mathrm{CDM}$ cosmology with $H_{0}=73.24 \mathrm{~km} \mathrm{~s}^{-1} \mathrm{Mpc}^{-1}$ (Riess et al. 2016) and $\Omega_{m}=0.275$ (Amanullah et al. 2010).

\section{The ZTF-2018 High-cadence Sample of SNe Ia}

\subsection{Observations}

The ZTF camera is mounted on the 48 inch Samuel Oschin Telescope (P48) at Palomar Observatory (Dekany et al. 2016). At a limiting magnitude of $r \sim 20.5 \mathrm{mag}$, three custom filters $\left(g_{\mathrm{ZTF}}, r_{\mathrm{ZTF}}\right.$, and $i_{\mathrm{ZTF}}$; hereafter $g, r$, and $i$ ) are designed to maximize throughput by avoiding major skylines at Palomar (Bellm et al. 2019b). ZTF divides its observing time between public surveys (40\%), partnership surveys (40\%), and Caltech surveys (20\%). Bellm et al. (2019a) provide details of the ZTF surveys. In brief, $85 \%$ of the public time was allocated to a "Northern Sky Survey" with a cadence of three days in $g$ and $r$, and the remaining $15 \%$ to a "Galactic Plane Survey" with two visits to the Galactic plane (one $g+$ one $r$ ) every night. The bulk of the partnership time in 2018 (May-December) was dedicated to two experiments, including an extragalactic highcadence experiment covering $\sim 2500 \mathrm{deg}^{2}$ with six visits (three $g+$ three $r$ ) every night, and a lower-cadence, widefield $i$-band survey. The Caltech time was conducted in a oneday cadence in both $g$ and $r$ with a total footprint of $\sim 3000 \mathrm{deg}^{2}$. Each night's schedule is arranged by the survey scheduler (Bellm et al. 2019a) to optimize volumetric survey speed (Bellm 2016). In this work, we only focus on the $g$ - and $r$-band observations within the partnership high-cadence fields.
The current ZTF alert distribution system (Patterson et al. 2019) generates a source packet once a transient ${ }^{23}$ is detected. By definition, a "detection" means that the observed flux is five times larger than the flux uncertainty (see Masci et al. 2019, Section 6). For each transient the alert packet includes a rolling 30 days history of detections and non-detections.

Following the association of all alerts generated at the same position, the GROWTH "Marshal" (Kasliwal et al. 2019) compiles a complete historical record of variability, which is further used to aggregate and visualize follow-up observations.

\subsection{Initial Sample Selection}

The sample selection process is summarized in Table 1. In total, there were 336 SNe Ia classified in the partnership fields in 2018, 247 of which were observed as part of the high-cadence partnership survey. ${ }^{24}$ For the sample of $247 \mathrm{SNe}$ with highcadence observations, we performed a preliminary fit to their light curves using the SALT2 software package (Guy et al. 2007) implemented in the sncosmo Python package (Barbary et al. 2016) ${ }^{25}$ to estimate the time of maximum light. The sample was further reduced to include only those sources with more than one detection in either the $g$ or $r$ band obtained at least five days before the SALT2-estimated time of $B$-band maximum, $t_{B, \max }$. This resulted in a selection of $191 \mathrm{SNe}$.

Note that although the 191 SNe were all discovered in the partnership high-cadence fields, some of them also have observations during the public or Caltech time. We retained those observations in the following analysis.

The ZTF Science Data System (ZSDS) constructed reference images for each field and filter by taking the stack-average of 15-40 historical images ${ }^{26}$ (Masci et al. 2019). Observations for each target could be covered by multiple fields. To ensure that the reference images do not contain contamination from SN flux, we need to treat each field (with specific CCD-quadrant therein) for a given filter separately. Hereafter we use "fcqf ID," defined by

$$
\begin{aligned}
(\text { fcqf ID })= & (\text { field ID }) \times 10000+(\text { CCD ID }) \times 100 \\
& +(\text { quadrant ID }) \times 10+(\text { filter ID })
\end{aligned}
$$

as an identifier of the reference images.

We grouped observations by fcqf ID, and excluded those where the time of the latest exposure used to create the reference product was within 25 days of $t_{B, \max }$ (in the $\mathrm{SN}$ rest frame). Since the typical rise time of SNe Ia is 17 days (Firth et al. 2015), 25 days is a conservative choice. For each target, we further required that the remaining number of observations in both $g$ and $r$ must be no less than 35. $154 \mathrm{SNe}$ met this criterion.

\section{Data Analysis}

We perform "forced" point-spread function (PSF) photometry to extract precise flux measurements of the SN in all ZTF images, including those that were obtained prior to explosion. Forced PSF light curves are obtained by measuring the PSF flux at the position of the $\mathrm{SN}$ in all epochs. Figure 1 demonstrates the difference between the light curves generated by the ZTF alert packets and forced-PSF photometry. The improvement is clear as highlighted

\footnotetext{
23 For the purpose of this paper, moving objects are ignored.

24 A measurement of the detection efficiency and completeness of this sample is beyond the scope of this study and will be addressed in a future paper (J. Nordin et al. 2019, in preparation).

25 https://sncosmo.readthedocs.io/en/v2.0.x/models.html

26 The ZTF camera has 16 CCDs, with each CCD divided into four quadrants.
} 
Table 1

Steps in Sample Selection

\begin{tabular}{lll}
\hline \hline Step & Criteria & Total \# SNe \\
\hline 1 & Spectroscopically classified SNe Ia observed by the ZTF partnership survey & 336 \\
2 & Observed by the high-cadence fields & 247 \\
3 & At least one detection on the Marshal light curve earlier than 5 days prior to $t_{B, \max }$ & 191 \\
4 & Remove observations where the reference images are obtained after $t_{B, \max }-25$ days & \multicolumn{1}{c}{ Extract forced PSF photometry light curves } \\
\hline 5 & & \multicolumn{1}{c}{ Before $t_{B, \max }$, the target must be detected in both $g$ and $r$ over at least five nights } \\
6 & The first $3 \sigma$ detection in both $g$ and $r$ must be earlier than $t_{B, \max }-10(1+z)$ & 140 \\
7 & Must be detected at least once in both $g$ and $r$ in $\left[t_{B, \max }, t_{B, \max }+20(1+z)\right]$ & 129 \\
\hline
\end{tabular}

by the dotted box, where the forced photometry recovers detections that are otherwise missed by the real-time pipeline. It is also the case that the forced photometry provides deeper pre-explosion upper limits. Thus, forced-PSF photometry can (i) provide sub-threshold flux measurements, (ii) reveal structure in the early-time light curves, and (iii) allow more stringent constraints to be placed on the epoch of explosion.

\subsection{Astrometry}

The position of ZTF transients reported on the GROWTH Marshal is based on the initial detection of the source, which is often at low signal-to-noise ratio $(\mathrm{S} / \mathrm{N})$. As the first step of forced-PSF photometry, we need to determine the position of the transient more accurately. To this end, for each SN, we obtained the coordinates in all epochs where the $\mathrm{SN}$ is detected using Kowalski, ${ }^{27}$ a ZTF database system. The typical scatter in both R.A. and decl. is $\sim 0$ ". 088 (0.09 pixel size). This uncertainty is small, and thus we do not incorporate it into the PSF modeling. We took the median R.A. and decl. as the true position of each SN.

\subsection{Data Description}

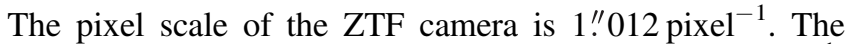
typical seeing-limited FWHM of the PSF is $\sim 2^{\prime \prime} \mathrm{pixel}^{-1}$. Figure 2 shows an example of the point-source cutouts of a difference image and its normalized PSF-model template. The PSF is normalized such that the sum of all pixel response values is equal to 1 . These images are available at the NASA/ IPAC Infrared Science Archive (IRSA). ${ }^{28}$

The difference image PSF is a product of the ZOGY image subtraction algorithm (Zackay et al. 2016). ZOGY generates this by combining the input PSF templates from the science and reference images prior to subtraction. The science and reference image PSF templates were generated using an automated version of the classic DAOPhot/AllStar software (Stetson 1987), with further optimizations for ZTF (Masci et al. 2019; Sections 3.5 and 4). A linearly spatially varying PSF model consisting of a Gaussian core modulated by corrections is fit to a set of pre-filtered (uncontaminated and unsaturated) stars in the science and reference images separately. Only PSF estimates at the center of the science and reference CCDquadrants are used for input to ZOGY. Prior to use in ZOGY, the PSF templates are further regularized to suppress pixel outliers in their outer regions. ZOGY then combines the PSFs using a Fourier inversion method to generate a single PSF

\footnotetext{
27 https://github.com/dmitryduev/kowalski

28 https://irsa.ipac.caltech.edu
}

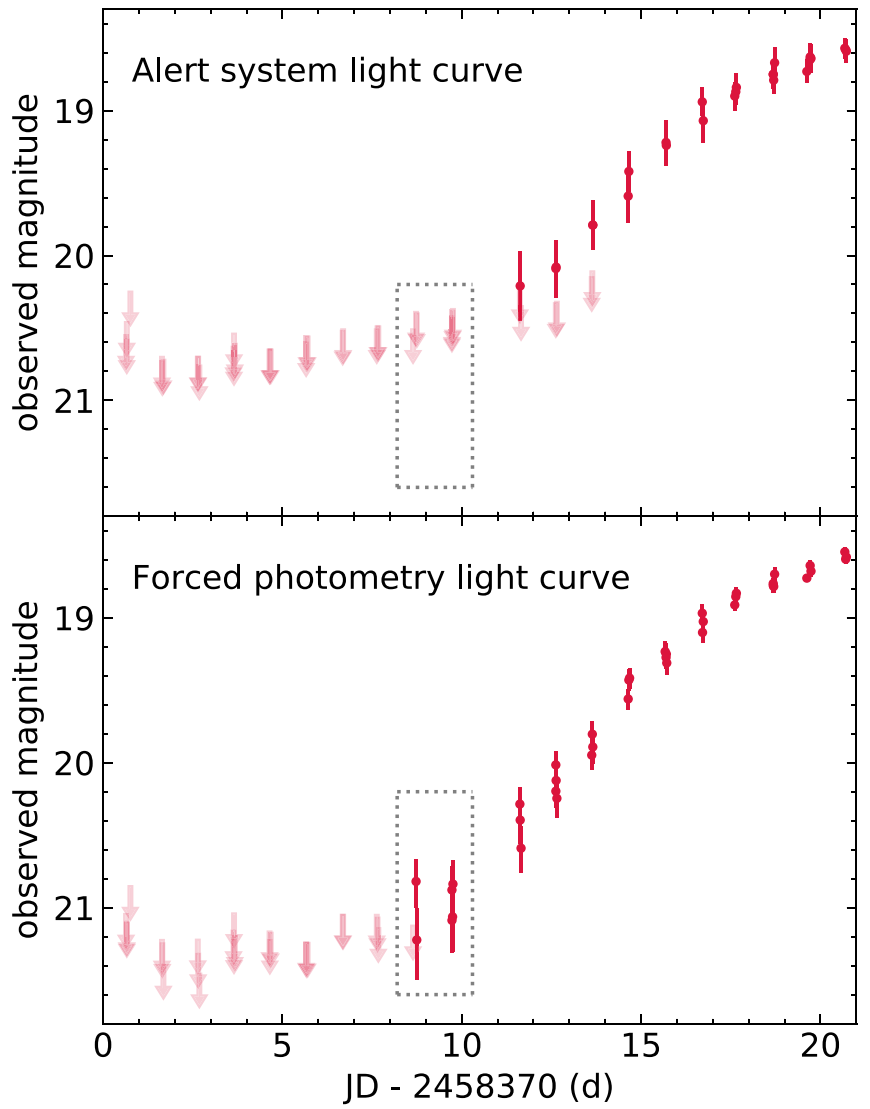

Figure 1. Upper panel: the $r$-band light curve of ZTF18abxxssh generated by the alert distribution system. Bottom panel: forced-PSF photometry light curve of the same object. The dotted box highlights additional early-time $r$-band detections recovered by forced photometry.

template for the difference image. This single PSF therefore represents an effective PSF for the entire quadrant image. Its spatial variation on quadrant scales (inherent in the science and reference images) is $<1 \%$. This is not significant enough to impact the accuracy of our PSF-fit photometry in our magnitude range of interest ( $~ 17 \mathrm{mag}$ ), where measurements are dominated by sky background noise.

Hereafter we denote the pixel values of model image and difference image by $x_{i}$ and $y_{i}^{\prime}$, respectively. $x$ is unitless and $y^{\prime}$ has the unit of detector data number (DN), which is analogous to analog digital units (ADU). We estimate the background noise $\sigma_{\text {bkg }}$ (in the unit of DN) from all pixels inside an annulus centered at the location of the target, with an inner radius $r_{\text {in }}=10$ pixels and an outer radius $r_{\text {out }}=15$ pixels (indicated by the dashed 

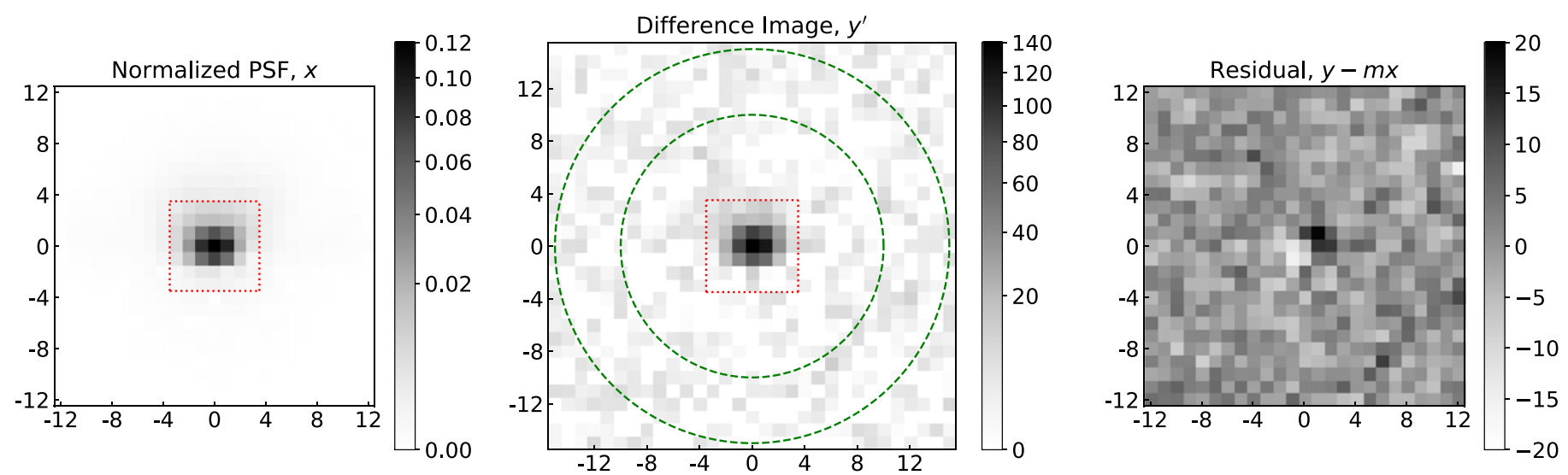

Figure 2. An example of the cutouts of the PSF-model image (left, $25 \times 25$ pixels), the difference image (middle, $31 \times 31$ pixels), and the residual (right, $25 \times 25$ pixels) centered on the position of the target. The central $7 \times 7$ pixel cutouts are marked by the dotted red squares. The background region is marked by the dashed green annulus, with inner radius $=10$ pixels and outer radius $=15$ pixels. Note that 10 pixels $\approx 5$ FWHM.

green circles in Figure 2). Thus, $\sigma_{\mathrm{bkg}}=0.5 \times[$ (the 84 th percentile of $\left.y_{b k g}^{\prime}\right)$ - (the 16th percentile of $y_{b k g}^{\prime}$ )]. Ideally the median of all pixels in this background annulus should be around zero, i.e., median $\left(y_{\text {bkg }}^{\prime}\right) \approx 0$, assuming that image subtraction is perfect. However, it was found that the background level is sometimes far from zero in regions close to the center of galaxies. Therefore, we subtracted the local background from $y^{\prime}$ to get a more robust estimate of the excess flux relative to background: $y_{i}=y_{i}^{\prime}-\operatorname{median}\left(y_{\mathrm{bkg}}^{\prime}\right)$. The $y$ thus derived also has units of DN.

\subsection{The PSF Fitting Method}

In ZSDS, image subtraction is performed using the ZOGY algorithm (Zackay et al. 2016). The PSF template image (left panel of Figure 2) is generated such that the difference image (right panel of Figure 2) can be modeled by the PSF image multiplied by a number $m$, plus some random noise $\epsilon$, i.e., $y=m x+\epsilon$. Here, $m$ is the PSF-fit flux in the unit of DN, and $\epsilon$ is a noise term: $\epsilon \sim \mathcal{N}\left(0, \sigma^{2}\right)$. The statistical pixel uncertainty for $y_{i}$ is

$$
\sigma_{i}^{2}=\frac{y_{i}}{\text { gain }}+\sigma_{\mathrm{bkg}}^{2}
$$

where the gain is the electronic detector-gain (in the unit of electron per DN).

Although our task is simply to fit a straight line to a set of $\left(x_{i}\right.$, $y_{i}$ ) pairs, there is no consensus on how to derive the best measurement of $m$ (see Hogg et al. 2010 or Sharma 2017 (Section 2) for a recipe on this problem). The commonly adopted maximum likelihood estimate has the advantage of being fast, but is only optimal for the background-dominatednoise limit (Zackay et al. 2016). In principle we expect measurements of intra-night observations to be consistent with each other, but we found that our initially adopted maximum likelihood method did not provide such a result. Instead, a Bayesian method was attempted whereby we implemented a Markov chain Monte Carlo (MCMC) fit, which was found to give the smallest variance of intra-night observations in the same band. Therefore, we adopted the MCMC approach, and utilized emcee, which is an affine-invariant MCMC ensemble sampler that uses multiple walkers to sample the posterior probability distribution (Goodman \& Weare 2010; ForemanMackey et al. 2013).

Assuming that the uncertainties in Equation (2) are underestimated by a constant systematic factor $\sigma_{0}$, the probability of $y_{i}$ given $\left(x_{i}, \sigma_{i}, m, \sigma_{0}\right)$ is

$$
\begin{aligned}
& p\left(y_{i} \mid m, \sigma_{0}, x_{i}, \sigma_{i}\right) \\
& =\frac{1}{\sqrt{2 \pi\left(\sigma_{i}^{2}+\sigma_{0}^{2}\right)}} \exp \left(-\frac{\left(y_{i}-m x_{i}\right)^{2}}{2\left(\sigma_{i}^{2}+\sigma_{0}^{2}\right)}\right) .
\end{aligned}
$$

From (3) it follows that the log-likelihood is

$$
\ln \mathcal{L}=\sum_{i}^{N}\left[\ln \left(\frac{1}{\sqrt{2 \pi\left(\sigma_{i}^{2}+\sigma_{0}^{2}\right)}}\right)-\left(\frac{y_{i}-m x_{i}}{2\left(\sigma_{i}^{2}+\sigma_{0}^{2}\right)}\right)^{2}\right] .
$$

We only include the central $7 \times 7$ cutout (indicated by the dotted red square in Figure 2) in the fit, so $N=49$ is the number of pixels that were taken into consideration.

The posterior probability distribution function of the model parameters $\left(m, \sigma_{0}\right)$ for each observation can be obtained from the following equation according to Bayes' theorem:

$$
\begin{aligned}
p & \left(m, \sigma_{0} \mid\left\{y_{i}\right\}_{i=1}^{N}, x_{i}, \sigma_{i}\right) \\
& =\frac{1}{Z} p\left(\left\{y_{i}\right\}_{i=1}^{N} \mid m, \sigma_{0}, x_{i}, \sigma_{i}\right) p\left(m, \sigma_{0}\right)
\end{aligned}
$$

where $Z$ is a normalization factor and $p\left(m, \sigma_{0}\right)$ is the prior.

We adopted wide and flat priors: (i) $m$ was uniformly distributed in the range $\left[-10^{6}, 10^{6}\right]$; (ii) $\sigma_{0}$ was logarithmically uniformly distributed in the range $\left[e^{-10}, e^{10}\right]$. The twodimensional parameter space was investigated using 250 walkers. All models were run to convergence as determined by the evolution of the autocorrelation of the individual MCMC chains (see https://emcee.readthedocs.io/en/latest/ tutorials/autocorr/). A demonstration of this step is given in Figure 3.

We obtained the posterior probability distributions for $m$ and $\sigma_{0}$ as the output from the MCMC fitting, and marginalized over $\sigma_{0}$ to estimate the slope, $m$. Throughout this paper, we take the median value of the distribution as the measured flux, $f_{\mathrm{mcmc}}$, whereas the uncertainty on this value, $\sigma_{f_{\text {mcmc }}}$, was estimated as half of the difference between the 84th and 16th percentiles of 


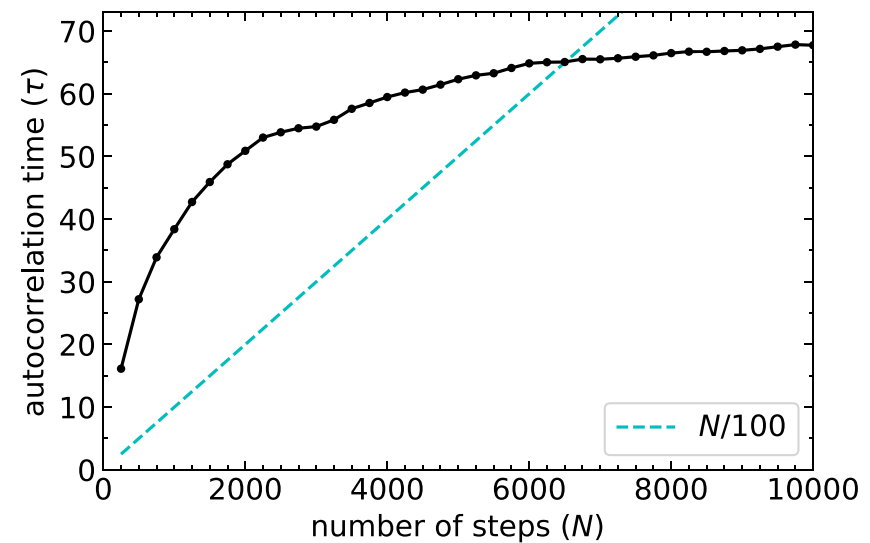

Figure 3. The solid black line shows autocorrelation time $(\tau)$ as a function of sample step $(N)$. We compute $\tau$ every 250 steps, and the MCMC chains are stopped when the difference of two consecutive $\tau$ values is less than 0.01 . In the case shown in this figure, the MCMC chains are converged after 10,000 steps. The dashed cyan line intercepts with the black line at $N \approx 6500$. When $N>6500$, the effective number of samples is larger than 100 .

the marginalized posterior of $m$. Both $f_{\mathrm{mcmc}}$ and $\sigma_{f_{\mathrm{mcmc}}}$ have units of DN.

\subsection{Quality Filtering}

A small fraction of the ZTF data were acquired through intermittent cloud cover or featured extremely high backgrounds due to the proximity of the full moon. This affects the resulting photometric calibration. Therefore, several cuts were applied to ensure the quality of our photometric measurements.

1. We removed data points with non-zero values of infobits. This keyword is a 16-bit integer that encodes the status of processing and instrumental calibration steps for the science image; specific operations that fail to meet predefined quality criteria are assigned to individual bits [0..15]. These bits can be "AND'ed" with a template bit-string to reject science images that failed specific calibration steps.

2. We removed data points with scisigpix $>25$. scisigpix is a robust estimate of spatial noise-sigma per pixel in the input science image; this is based on half the "84.13 - 15.86" percentile difference in pixel values.

3 . We removed data points with seeing $>4^{\prime \prime}$, where seeing captures the FWHM of the point source.

4. We removed the observation if there was any pixel in the central $7 \times 7$ cutout with $y_{i}<-500$. Typically, pixels with negative values of several hundreds are caused by saturation in the reference image, and thus should be removed in the fitting. It is difficult to define a threshold to mask out those bad pixels, so we choose to remove the observations instead.

infobits and seeing are in the header of every science image product in the archive as well as in IRSA's science image metadata database (DB) table. However, scisigpix is internal and not propagated to any publicly visible product, but it can be estimated directly from the science images.

Note that while alternative prescriptions to flag observations may be adopted, we found the above cuts to be adequate to remove most of the non-photometric data in our sample. The flagged "bad" observations are not included in the table of final light curves accompanying this paper.

\subsection{Baseline Correction}

A baseline correction was applied to $f_{\text {mcmc }}$ to correct for any residual offset in the "history" of the light curve. We chose to define any data earlier than $T_{\text {before }}$ days prior to $t_{B \text {, max }}$ (in the rest frame) to be the "history" where $T_{\text {before }}=20$. We visually inspected the light curves to make sure that no supernova flux was included in the baseline. For six targets (ZTF18aaykjei, ZTF18abhpgje, ZTF18abdpvnd, ZTF18aaytovs, ZTF18abddmrf, and ZTF18aawpcel) where the rest-frame rise time is obviously longer than 20 days, we adjusted the value of $T_{\text {before }}$ to 25 . Note that these objects are peculiar $\mathrm{SNe}$ with longer rise time than normal SNe Ia (see Table 2 and Section 5). Since the reference images for different fcqf ID (Equation (1)) were created by different observations, the baseline level should be determined separately for every possible combination of field and filter. For example, Figure 4 shows the light curve of ZTF18aazblzy, a normal SN Ia in our sample at redshift $z=0.0653$. The lower panels show the number of observations in each fcqf ID in the baseline region $\left(N_{\text {base }}\right)$, the offset level $C$, as well as the reduced chi-square statistic $\left(\chi_{\nu}^{2}\right)$ :

$$
\chi_{\nu}^{2}=\frac{1}{\nu} \sum_{i=1}^{N_{\text {base }}} \frac{\left(C-f_{\mathrm{mcmc}, i}\right)^{2}}{\sigma_{f_{\mathrm{mcmc}}, i}^{2}}
$$

where $\nu=N_{\text {base }}-1$ is the degree of freedom and $C$ is calculated as the weighted mean of all $f_{\text {mcmc }}$ measurements in the baseline.

Figure 5 shows the distribution of $\chi_{\nu}^{2}$ versus $C$ for all targets in our sample. Although we may expect $C \approx 0$, a non-zero historical baseline level can occur if

(i) The reference image is contaminated by residual flux from the actual transient being measured, i.e., the input images used to construct the reference inadvertently included epochs containing significant transient flux. (This is unlikely because we applied selection step 4 in Table 1.)

(ii) The reference image is contaminated by an instrumental artifact that was not properly masked (or detected as an outlier) prior to co-addition.

(iii) There are systematic residuals from persistently inaccurate gain-matching between the science and reference images. This is usually triggered by imperfect flat-fielding of the science images used to construct the reference image, i.e., the reference image exhibits a spatial variation in its photometric gain. This systematic spatial variation will persist (be imprinted) in all subtraction images constructed using this reference image. If the gain-mismatch between science and reference images at the location of the transient is significant, this will also lead to inflated $\chi_{\nu}^{2}$ values since measurements relative to the baseline will be noisier (inflated by a hidden systematic gain factor) than those represented by the individual-epoch measurement uncertainties $\left(\sigma_{f_{\mathrm{mcmc}}, i}\right)$.

A sufficient number of historical measurements is required for robust estimates of $C$ and $\chi_{\nu}^{2}$. Large absolute values of $C$ or large values of $\chi_{\nu}^{2}$ should be considered as "red flags" suggesting further analysis and visual examination of the images, particularly the reference image to search for the systematic described in case (iii) above. We mitigate this systematic by subtracting the baseline $C$ from the measured $f_{\text {mcmc }}$, and if $\chi_{\nu}^{2}>1$ we multiply the raw $\sigma_{f_{\text {mcm }}}$ by $\sqrt{\chi_{\nu}^{2}}$. The 
Table 2

General Information on $127 \mathrm{SNe}$ Ia

\begin{tabular}{|c|c|c|c|c|c|c|c|}
\hline $\begin{array}{l}\text { ZTF Name } \\
(\mathrm{ZTF} 18) \\
(1)\end{array}$ & $\begin{array}{l}\text { R.A. (J2000) } \\
\text { (deg) } \\
(2)\end{array}$ & $\begin{array}{c}\text { Decl. (J2000) } \\
\text { (deg) } \\
(3)\end{array}$ & $\begin{array}{l}\text { IAU } \\
\text { Name } \\
(4)\end{array}$ & $\begin{array}{l}\text { TNS Internal } \\
\text { Name } \\
(5)\end{array}$ & $\begin{array}{c}\text { Telescope } \\
\text { (6) }\end{array}$ & $\begin{array}{c}\text { Spectral Phase } \\
\text { (7) }\end{array}$ & $\begin{array}{l}\text { Ia Subtype } \\
\text { (8) }\end{array}$ \\
\hline aansqun & 251.2920505 & 42.7178671 & SN2018dyp & ZTF18aansqun & P60 & -1 & normal \\
\hline aaqcozd & 190.5630550 & 42.2717146 & SN2018bjc & ATLAS18odd & NOT & -4 & normal \\
\hline aaqqoqs & 207.9703694 & 47.2569927 & SN2018cbh & ZTF18aaqqoqs & P200 & -6 & 99aa-like \\
\hline aawpcel & 195.3210357 & 59.8100342 & SN2018cir & ZTF18aawpcel & P60 & +1 & $\mathrm{SC} *$ \\
\hline aaytovs & 266.4724817 & 31.7105165 & SN2018crk & ZTF18aaytovs & P200 & +14 & 99aa-like \\
\hline aazblzy & 242.8394794 & 36.9943003 & SN2018cri & ZTF18aazblzy & P200 & -10 & normal \\
\hline abauprj & 254.7709972 & 47.2364390 & SN2018cnw & ZTF18abauprj & NOT & -6 & 99aa-like \\
\hline abclfee & 258.5925379 & 48.2643039 & SN2018cxk & ZTF18abclfee & P60, LT, P200, P60, P200 & $+2,+4,+8,+8,+35$ & 02cx-like \\
\hline abddmrf & 226.9673020 & 38.0484486 & SN2018dsx & ATLAS18sdi & P200 & +36 & $\mathrm{SC} *$ \\
\hline abdmgab & 250.9022676 & 33.5336160 & SN20181ph & ZTF18abdmgab & Keck I & +1 & 86G-like \\
\hline abuqugw & 244.5576659 & 39.1238139 & SN2018geo & ATLAS18vca & P60 & -9 & normal \\
\hline
\end{tabular}

Note. Column (4): SN IAU name from TNS. Column (5): TNS internal name (indicating the discovery group). Columns (6) and (7): follow-up telescope and epoch of the spectrum with respect to $t_{g, \max }$ used to determine the spectral subtype. $t_{g, \max }$ is the epoch of $g$-band maximum light provided in Table 3 and calculated in Section 4.3. Column (8): if classification cannot be reliably determined from spectroscopy alone, the subtype ends with an asterisk. See Section 4.2 for details.

(This table is available in its entirety in machine-readable form.)

photometric uncertainties thus derived should be considered as a conservative estimate. For others who would like to model these light curves in the future, it is also advised to perform such a baseline validation and uncertainty scaling, or to remove observations associated with $\chi_{\nu}^{2} \gtrsim 4$ or $|C| \gtrsim 15$ from the sample. In the light curves accompanying this paper we provide our measurements of $C$ and $\sqrt{\chi_{\nu}^{2}}$, and set the values of these columns to -999 if the corresponding fcqf ID has $N_{\text {base }}<30$.

With the knowledge of the zero-point magnitude (zp) of the difference image provided by ZSDS, the zero-point flux in the unit of DN $\left(f_{0}\right)$ can be calculated:

$$
\begin{gathered}
f_{0}=10^{0.4 \times \mathrm{zp}} \\
\sigma_{f_{0}}=f_{0} \frac{\ln (10)}{2.5} \sigma_{\mathrm{zp} .}
\end{gathered}
$$

Thus, the dimensionless flux ratio is

$$
\begin{gathered}
f_{\text {ratio }}=f_{\text {mcmc }} / f_{0} \\
\sigma_{f_{\text {ratio }}}=\sqrt{\left(\frac{\sigma_{f_{\text {mcm }}}}{f_{0}}\right)^{2}+\left(\frac{f_{\mathrm{mcmc}} \sigma_{f_{0}}}{f_{0}^{2}}\right)^{2}} .
\end{gathered}
$$

We define the detection threshold of $\mathrm{S} / \mathrm{N}$ to be $3 .{ }^{29}$ That is to say, whenever $f_{\text {ratio }}>3 \times \sigma_{f_{\text {ratio }}}$, the conversion from flux to magnitude can be applied:

$$
\begin{gathered}
m=-2.5 \times \log f_{\text {ratio }} \\
\sigma_{m-}=2.5 \times \log \left(1+\sigma_{f_{\text {ratio }}} / f_{\text {ratio }}\right) \text { brighter end }
\end{gathered}
$$

\footnotetext{
${ }^{29}$ We follow the rationale illustrated in http://web.ipac.caltech.edu/staff/ fmasci/ztf/forcedphot.pdf to choose $\mathrm{S} / \mathrm{N}=3$ and use $5 \times \sigma_{f_{\text {ratio }}}$ to define upper limits.
}

$$
\sigma_{m+}=-2.5 \times \log \left(1-\sigma_{f_{\text {ratio }}} / f_{\text {ratio }}\right) \text { fainter end. }
$$

For non-detections, we compute $5 \sigma$ upper limits as

$$
m_{\text {lim }}=-2.5 \times \log \left(5 \times \sigma_{f_{\text {ratio }}}\right) .
$$

\subsection{Final Sample Selection}

With the 154 targets selected in Section 2.2, we further apply the cuts illustrated in steps 5-7 of Table 1. Steps 5 and 6 are made to ensure that targets included in our sample have both early-time detections and relatively dense light-curve sampling. The requirement in step 7 is to ensure that epochs of maximum light in $g$ and $r$ can be accurately estimated (in Section 4.3). In the end, $127 \mathrm{SNe}$ Ia were finally retained in our sample. Table 2 provides general information on these targets. Table 3 summarizes additional photometric properties of this sample, and Table 5 provides their forced-PSF photometry light curves. Photometric and spectroscopic observations of sources rejected by our sample selection criteria will be published in a separate study on the full sample of SNe Ia found by ZTF.

\section{Sample Properties}

We use ZTF transient names throughout this paper. In some cases these events were first reported by other groups (see Column (5) of Table 2), although the first detection in their forced-photometry light curves may precede the time of announcement on the Transient Name Server (TNS).

\subsection{Comparison with Previous Samples}

The redshift distribution of all targets in our sample is plotted in Figure 6. Shown in black are $46 \mathrm{SNe}$ with host-galaxy redshifts from the NASA/IPAC Extragalactic Database (NED), 

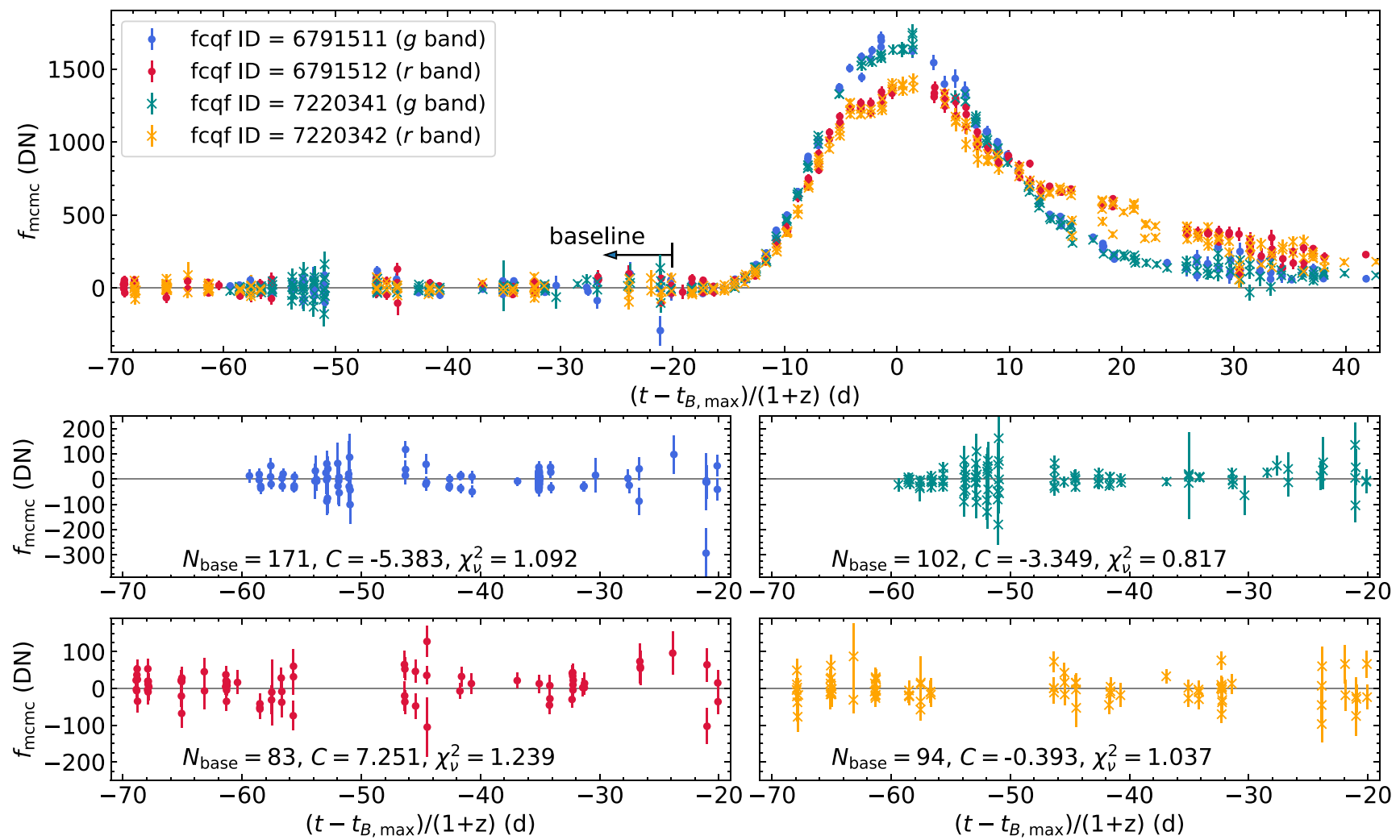

Figure 4. Upper panel: P48 light curve of ZTF18aazblzy. This target was observed in two filters ( $g$ and $r$ ) and two fields (fields 679 and 722 ). Observations associated with different fcqf ID (Equation (1)) are shown in distinctive colors. The $x$-axis shows time measured in rest-frame days relative to the SALT2-estimated $B$-band maximum epoch $\left(t_{B, \max }\right)$. Lower four panels: a zoom-in of the baseline region. The number of baseline observations $\left(N_{\text {base }}\right)$, the calculated offset level $(C)$, and the reduced chi-square $\left(\chi_{\nu}^{2}\right)$ for each fcqf ID are shown in each panel.

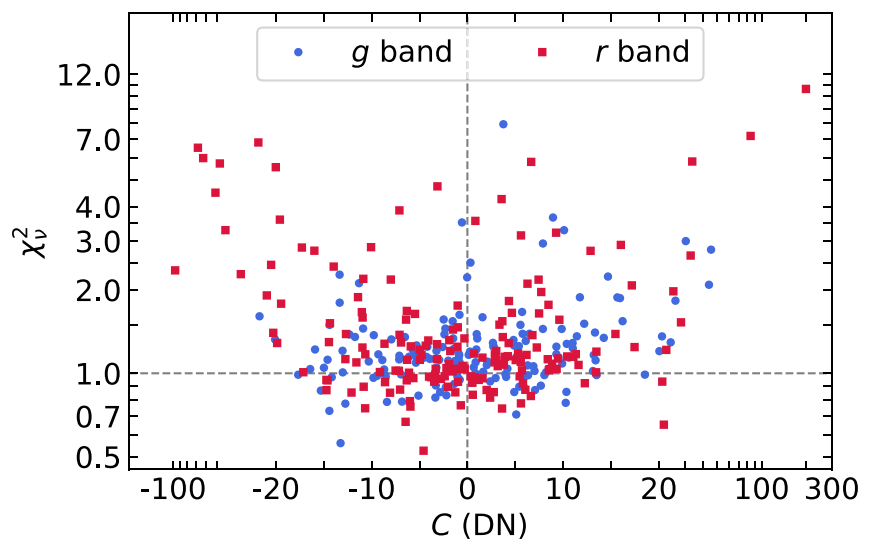

Figure 5. Distribution of $\chi_{\nu}^{2}$ and $C$ color-coded by filters $(g$ and $r$ ). Note that the horizontal axis is shown with a linear scale for $-20<C<20$ and a $\log$ scale for $|C|>20$. The vertical axis is shown on a $\log$ scale. The dashed horizontal line indicates $\chi_{\nu}^{2}=1$ and the dashed vertical line indicates $C=0$. The median of $C$ is -1.49 , and the median of $\chi_{\nu}^{2}$ is 1.21 .

while redshifts of the 16 shown in gray are measured from host-galaxy lines in the $\mathrm{SN}$ spectrum or from a spectrum of the host galaxy itself. Redshifts of the remaining 65 targets shown in blue are inferred from the SN spectrum (see Section 4.2 for details). Redshifts estimated directly from the SN spectrum have a typical uncertainty of $\sim 0.004$ (Fremling et al. 2019). The 16th, 50th, and 84th percentiles of the redshift distribution are $z=0.051,0.074$, and 0.106 .

We summarize early-time photometric samples of SNe Ia with low to intermediate redshift from multiple surveys in
Table 4. The table is divided into two, with the top half listing surveys focused on the follow-up of SNe Ia, while the bottom half lists surveys that both discover and follow up SNe Ia. We use this split to better highlight the number of SNe with early observations, because these numbers are not directly comparable between surveys that discover $\mathrm{SNe}$ and those that only perform follow-up observations. Hereafter we only compare the ZTF sample with the LOSS, SDSS-II, iPTF/PTF, and TESS samples.

Figure 7 shows the distribution of first-detection epoch relative to $B$ - or $g$-band maximum light for $\mathrm{ZTF}, \mathrm{PTF} / \mathrm{iPTF}$, SDSS, and LOSS. SNe with no pre-maximum detections are not shown in the figure. Figure 8 shows the histogram of the number of nights that each target was observed (upper limits are not included, intra-night observations are counted as one night). The TESS-2018 sample is not plotted because it has a relatively small size. We note that Column (5) of Table 4 may not be appropriate for this sample, since TESS is a space satellite that provides light curves with a cadence of 30 minutes (Ricker et al. 2015). Among the 18 TESS SNe, seven were observed in two sectors and 11 were covered in one sector (27 days per sector). Ten events were detected at least 14 days prior to maximum light (see Figure 1 of Fausnaugh et al. 2019).

LOSS is a targeted survey that uses the Katzman Automatic Imaging Telescope (KAIT, Filippenko et al. 2001; Li et al. 2003) as its discovery engine. As shown in Figure 7, this sample contains the smallest number of events observed prior to -12 days, likely as a result of KAIT's relatively small aperture $\left(m_{\lim } \approx 19 \mathrm{mag}\right)$ and slower cadence ( $\sim 3.5$ days $)$. 
Table 3

Photometric Properties of 127 SNe Ia

\begin{tabular}{|c|c|c|c|c|c|c|}
\hline $\begin{array}{l}\text { ZTF Name } \\
\text { (ZTF18) } \\
(1)\end{array}$ & $\begin{array}{c}\text { Redshift } \\
\text { (2) }\end{array}$ & $\begin{array}{c}E(B-V) \\
(\mathrm{mag}) \\
(3)\end{array}$ & $\begin{array}{c}t_{g, \max } \\
(\mathrm{MJD}) \\
(4)\end{array}$ & $\begin{array}{c}\Delta m_{15}(g) \\
(\mathrm{mag}) \\
(5)\end{array}$ & $\begin{array}{c}\text { SALT2 } x_{1} \\
\text { (6) }\end{array}$ & $\begin{array}{c}\text { SALT2 } c \\
\text { (7) }\end{array}$ \\
\hline aansqun & 0.0597 & 0.0127 & $58313.56 \pm 0.18$ & $0.99 \pm 0.08$ & $-1.72 \pm 0.61$ & $0.20 \pm 0.10$ \\
\hline aaqcozd & 0.0732 & 0.0203 & $58253.02 \pm 0.06$ & $0.96 \pm 0.02$ & $-1.23 \pm 0.07$ & $-0.10 \pm 0.01$ \\
\hline aaqqoqs & 0.082 & 0.0137 & $58261.21 \pm 0.03$ & $0.68 \pm 0.03$ & $1.22 \pm 0.27$ & $-0.01 \pm 0.03$ \\
\hline aawpcel & 0.151 & 0.0108 & $58279.48 \pm 1.43$ & $0.53 \pm 0.09$ & $3.23 \pm 0.78$ & $0.12 \pm 0.06$ \\
\hline aaytovs & 0.0746 & 0.0523 & $58293.58 \pm 0.03$ & $0.59 \pm 0.03$ & $2.03 \pm 0.37$ & $0.12 \pm 0.04$ \\
\hline aazblzy & 0.0653 & 0.0116 & $58291.47 \pm 0.02$ & $1.02 \pm 0.02$ & $-1.68 \pm 0.09$ & $-0.07 \pm 0.02$ \\
\hline abauprj & 0.0242 & 0.0230 & $58301.59 \pm 0.02$ & $0.67 \pm 0.01$ & $1.34 \pm 0.04$ & $-0.01 \pm 0.01$ \\
\hline abclfee & 0.0290 & 0.0127 & $58299.13 \pm 0.16$ & $1.67 \pm 0.04$ & $-2.53 \pm 0.09$ & $0.19 \pm 0.02$ \\
\hline abddmrf & 0.163 & 0.0119 & $58307.57 \pm 1.02$ & $0.48 \pm 0.10$ & $2.53 \pm 0.93$ & $0.08 \pm 0.05$ \\
\hline abdmgab & 0.0803 & 0.0186 & $58310.98 \pm 0.18$ & $1.08 \pm 0.04$ & $-2.31 \pm 0.33$ & $0.12 \pm 0.05$ \\
\hline abuqugw & 0.0313 & 0.0064 & $58384.85 \pm 0.04$ & $\ldots$ & $-1.35 \pm 0.03$ & $-0.12 \pm 0.01$ \\
\hline
\end{tabular}

Note. Column (2): the host galaxy redshift ( $z$ ) is shown with four decimal places if (i) $z$ is taken from NED, (ii) $z$ is measured from the galaxy spectrum obtained by us, and (iii) $z$ is measured from an SN spectrum where the host $\mathrm{H} \alpha$ line can be identified. Redshifts inferred from an SNID fit on SN spectra are shown with three decimal places. The redshift of ZTF18abptsco was reported by ATel 12052 (Gomez et al. 2018) and was shown with two decimal places. Column (3): foreground Galactic extinction from Schlafly \& Finkbeiner (2011). Columns (4) and (5): values of $t_{g, \text { max }}$ and $\Delta m_{15}(g)$ for peculiar events (classified as "02cx-like," "Ia-CSM," "SC," or "SC*") were obtained from polynomial fits to the $g$-band light curve. For other objects, both $t_{g, \text { max }}$ and $\Delta m_{15}(g)$ were from SALT2 estimates. If a target does not have any $g$-band observations in the time range $\left[t_{g, \max }+10, t_{g, \max }+20\right], \Delta m_{15}(g)$ cannot be securely estimated and is thus shown blank (e.g., ZTF18abuqugw). Columns (6) and (7): the light-curve shape parameter $x_{1}$ and color $c$ from SALT2 fitting.

(This table is available in its entirety in machine-readable form.)

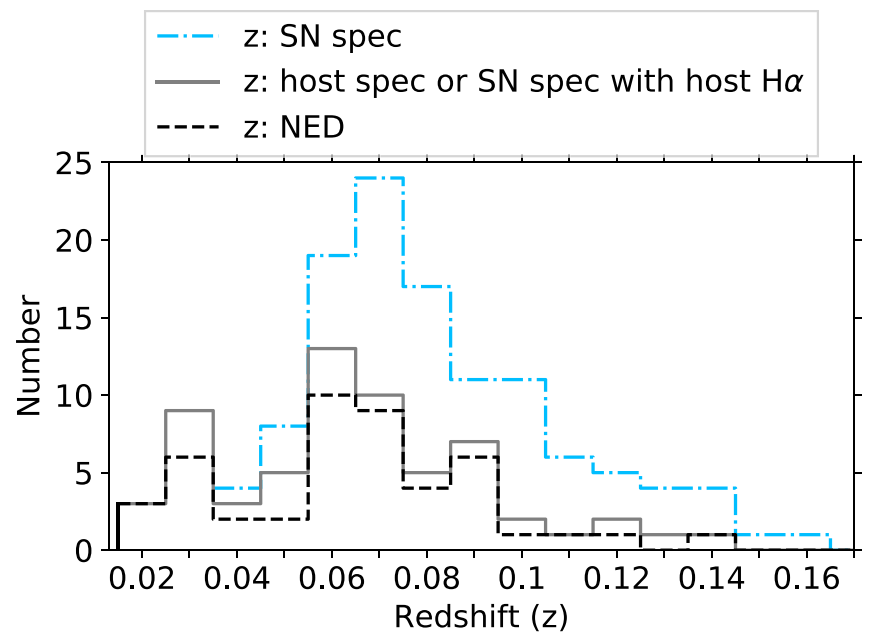

Figure 6. Redshift distribution of $127 \mathrm{SNe}$ Ia in our sample. Note that this a stacked histogram, such that the blue dotted-dashed line is the total distribution.

The SDSS-II survey provides one of the largest samples, which has a higher median redshift due to its large aperture $\left(m_{\mathrm{lim}} \approx 22.2 \mathrm{mag}\right)$. However, Figure 8 implies that the intervals between consecutive observations are relatively long due to the cadence of 4.5 days.

$\mathrm{PTF} / \mathrm{iPTF}$ observations were carried out at a variety of cadences between 1 and 4 days. Although the number of events with early-time photometry is comparable to that in the ZTF sample (Figure 7), only 27 (4) objects have more than 40 (60) nights of observations (Figure 8). Furthermore, the PTF/iPTF photometry is only in the $R$ band. In comparison, from the first 7 months of the ZTF high-cadence experiment, the number of objects with 40 or more nights of observation is 71 , and the number of objects with 60 or more is 35 . Only 11 targets were observed on fewer than 20 nights.

Among the $127 \mathrm{ZTF}$ SNe Ia in this study, 50 were discovered at least 14 days prior to $t_{B, \max }$, with nine being detected $>17$ days before $t_{B, \max }$. Among the latter nine events, three are peculiar events with longer rise time than normal SNe Ia (ZTF18aaykjei, ZTF18abdpvnd, and ZTF18abhpgje; see Section 5 for details), four are at very low redshift (ZTF18aasdted, ZTF18abcflnz, ZTF18abfhryc, and ZTF18abauprj, all within $z=0.04$ ), and two have possible early-time flux excess (ZTF18abxxssh at $z=0.064$ and ZTF18aavrwhu at $z=0.062$, a detailed analysis of these two will be presented elsewhere). In short, it is the rich information contained in the multi-band, well-sampled, early light curves that distinguishes ZTF as a unique survey for the study of early-time SNe Ia.

\subsection{Subtype Classification}

For the majority of SNe Ia the width of the light curve is related to the peak luminosity (Phillips 1993). Smaller subclasses can be characterized by their peculiar spectroscopic and photometric properties. For example, the overluminous 91T-like events (Filippenko et al. 1992b) and 99aa-like events (Li et al. 2001) have distinct Fe III lines dominating their early spectra; while the subluminous 91bg-like events (Filippenko et al. 1992a) and 86G-like events (Phillips et al. 1987) display pronounced $\mathrm{Si}$ II and $\mathrm{Ti}$ II lines. Recent reviews of the observational characteristics and physical interpretation of 
Table 4

Samples of SNe Ia with Low to Intermediate Redshift

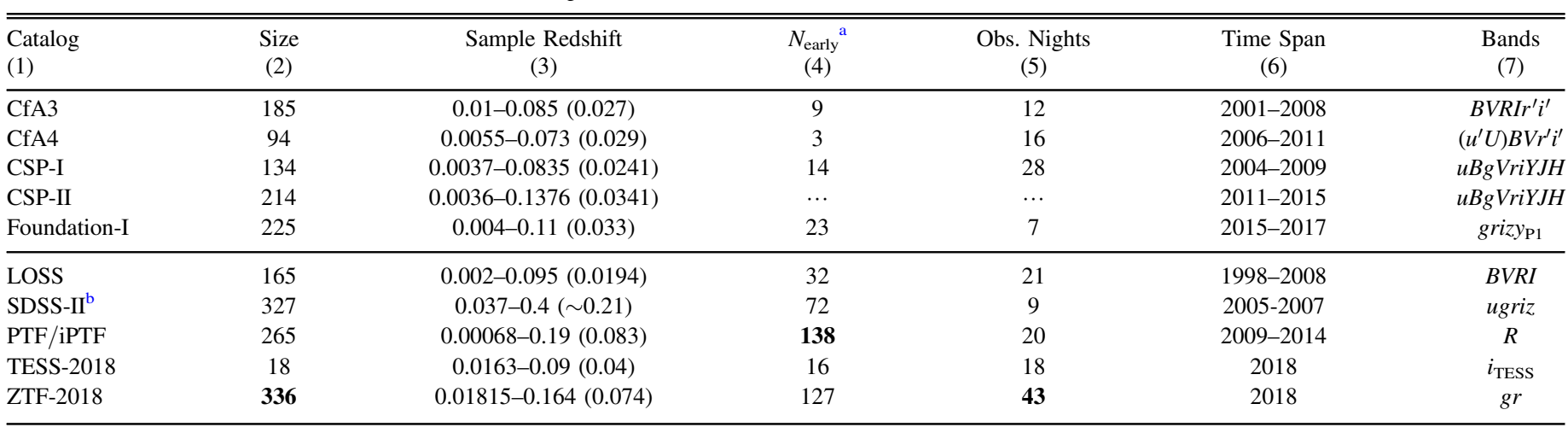

Notes. Column (2): total number of objects in each catalog. Column (3): redshift range with the median value shown in parenthesis. Column (4): number of targets with early observations. Column (5): median number of nights of observation per SN (upper limits not included). Column (6): survey period. Column (7): observing bands, those shown in parenthesis indicating that less than half of the targets were observed with the corresponding bands. Some estimates cannot be made due to our limited access to data. References: the Harvard-Smithsonian Center for Astrophysics SNe Ia sample (CfA3, Hicken et al. 2009; CfA4 Hicken et al. 2012), the Carnegie Supernova Project I (CSP-I) low-redshift sample (Hamuy et al. 2006; Contreras et al. 2010; Stritzinger et al. 2011; Krisciunas et al. 2017), CSP-II (Phillips et al. 2019), the Foundation Supernovae Survey data release I (Foley et al. 2018), the Sloan Digital Sky Survey II Supernova Survey (SDSS-II; Frieman et al. 2008), the Lick Observatory Supernova Search (LOSS, Filippenko et al. 2001) follow-up photometry program (Ganeshalingam et al. 2010), the (intermediate) Palomar Transient Factory (PTF/iPTF, Papadogiannakis et al. 2019), and SNe Ia observed in the first six sectors of the Transiting Exoplanet Survey Satellite (TESS) with pre-explosion observations (Fausnaugh et al. 2019).

${ }^{a}$ The number of SNe Ia with early observations, $N_{\text {early }}$, is defined as the number of targets with detections earlier than 10 days in the rest frame relative to the epoch of $B$-band peak luminosity. For SDSS-II this estimate is made relative to the time of $g$-band maximum light, while for the CfA3 and LOSS samples we use the observerframe phase.

${ }^{b}$ The size of the SDSS-II sample was reported to be 327 in Frieman et al. (2008), and increased to 500 later (Hayden et al. 2010a, 2010b). Sako et al. (2018) claimed 1364 SNe Ia from SDSS with spectroscopic redshifts (though some of these SNe were identified photometrically). The statistics shown for the SDSS-II sample are based on Frieman et al. (2008).

different subtypes can be found in the literature (e.g., Parrent et al. 2014; Maeda \& Terada 2016; Taubenberger 2017).

\subsubsection{Spectroscopic Observations}

A large fraction of our spectroscopic follow-up observations were conducted by the Spectral Energy Distribution Machine (SEDM, Blagorodnova et al. 2018; Rigault et al. 2019) on the robotic Palomar 60 inch telescope (P60, Cenko et al. 2006). Other follow-up instruments include the Double Spectrograph (DBSP; Oke \& Gunn 1982) on the Palomar 200 inch telescope (P200), the Low-Resolution Imaging Spectrometer (LRIS; Oke et al. 1995) on the Keck I $10 \mathrm{~m}$ telescope, the Andalucia Faint Object Spectrograph and Camera (ALFOSC) on the $2.56 \mathrm{~m}$ Nordic Optical Telescope (NOT), the Dual Imaging Spectrograph (DIS) on the Astrophysical Research Consortium (ARC) $3.5 \mathrm{~m}$ telescope at Apache Point Observatory (APO), the SPectrograph for the Rapid Acquisition of Transients (SPRAT) on the $2.0 \mathrm{~m}$ Liverpool Telescope (LT), and the Deveny spectrograph on the $4.3 \mathrm{~m}$ Discovery Channel Telescope (DCT; Bida et al. 2014).

Within our sample, 95 objects have one spectrum; 22 have two, five have three, one has four, two have five, and one has 10. The classification of ZTF18abptsco was reported via The Astronomer's Telegram (Gomez et al. 2018). If an object has more than one spectrum, we choose the one of highest $\mathrm{S} / \mathrm{N}$ or closest to maximum light for classification. The spectral phase for individual $\mathrm{SNe}$ is shown in Column (7) of Table 2. Figure 9 shows the distribution of rest-frame epoch relative to $g$-band maximum light (the estimation of $t_{g, \max }$ is illustrated in Section 4.3) used to determine the spectral subtype.
Within our sample, $92 \mathrm{SNe}$ are classified solely with SEDM spectra. These spectra will be described in detail in M. Rigault et al. (2019, in preparation) ${ }^{30}$ Below we describe our method for SN subtype classification. Table 6 provides information on 37 non-SEDM spectra of 34 targets, for which the subtype determination was not solely dependent on SEDM. ${ }^{31}$ A subset of these spectra are shown in Figure 10.

\subsubsection{Classification Based on SNID}

We use the Supernova Identification tool (SNID; Blondin \& Tonry 2007) to aid the determination of spectral subtype. SNID determines subtype via template-matching by cross-validation. It divides SNe Ia into five subtypes: Ia-norm, Ia-91T, Ia-91bg, Ia-csm, and Ia-pec. The template bank we used was from the original SNID templates-2.0 set, expanded with spectra of tidal disruption events (TDEs) and superluminous supernovae (SLSNe). An initial prior on template redshift was given if the host galaxy has an entry in NED. Otherwise we adopted the redshift determined by SNID as the SN redshift, which we report with less confidence in Table 2. If the top three best matched spectra returned by SNID were of the same subtype, then we considered this subtype as a reliable classification.

If the top three best matches returned by SNID were of different subtypes, we adopted the match where the inferred spectral phase (relative to maximum) was closest to the actual phase. If all matches were at similar phases, we further checked the absolute magnitude at maximum light. In cases where

\footnotetext{
${ }^{30}$ Most of the SEDM spectra are publicly available via TNS.

${ }^{31}$ Upon publication, these 37 spectra will be available in electronic format on the Weizmann Interactive Supernova Data Repository (WISEReP, Yaron \& Gal-Yam 2012).
} 
Table 5

P48 Photometry of 127 SNe Ia

\begin{tabular}{|c|c|c|c|c|c|c|c|c|c|c|c|c|}
\hline $\begin{array}{l}\text { ZTF Name } \\
\text { (ZTF18) } \\
\text { (1) }\end{array}$ & JD & $\begin{array}{c}\text { programid } \\
\text { (3) }\end{array}$ & fieldid & ccdid & qid & $\begin{array}{l}\text { filterid } \\
\text { (7) }\end{array}$ & $\begin{array}{c}\text { Seeing } \\
(\operatorname{arcsec}) \\
(8)\end{array}$ & $\begin{array}{c}\text { zp } \\
(\mathrm{mag}) \\
(9)\end{array}$ & $\begin{array}{c}\sigma_{\mathrm{zp}} \\
(\mathrm{mag}) \\
(10)\end{array}$ & $\begin{array}{c}f_{\mathrm{mcmc}} \\
(\mathrm{DN}) \\
(11)\end{array}$ & $\begin{array}{c}C \\
(\mathrm{DN}) \\
(12)\end{array}$ & $\begin{array}{l}\sqrt{\chi_{\nu}^{2}} \\
\text { (13) }\end{array}$ \\
\hline aazblzy & 2458291.7770833 & 2 & 722 & 3 & 4 & 2 & 2.164 & 26.185502 & $4.7941 \times 10^{-6}$ & $1361.0505270 \pm 37.7493381$ & -0.393 & 1.018 \\
\hline aazblzy & 2458291.7992593 & 2 & 679 & 15 & 1 & 2 & 2.240 & 26.170234 & $7.9630 \times 10^{-6}$ & $1367.4679899 \pm 36.4484999$ & 7.251 & 1.113 \\
\hline aazblzy & 2458291.7997338 & 2 & 722 & 3 & 4 & 2 & 2.057 & 26.179769 & $4.9365 \times 10^{-6}$ & $1377.8058619 \pm 33.4542310$ & -0.393 & 1.018 \\
\hline aazblzy & 2458291.8392708 & 2 & 722 & 3 & 4 & 1 & 1.970 & 26.274518 & $8.9916 \times 10^{-6}$ & $1630.3413509 \pm 35.3272129$ & -3.349 & 0.904 \\
\hline aazblzy & 2458292.7180556 & 2 & 722 & 3 & 4 & 1 & 2.103 & 26.299129 & $10.4533 \times 10^{-6}$ & $1634.6671366 \pm 51.8378991$ & -3.349 & 0.904 \\
\hline aaqcozd & 2458257.7669097 & 1 & 716 & 11 & 2 & 2 & 2.565 & 26.275000 & $15.3278 \times 10^{-6}$ & $1159.2163294 \pm 29.4045185$ & -0.981 & 0.965 \\
\hline aaqcozd & 2458257.7678472 & 2 & 716 & 11 & 2 & 2 & 2.554 & 26.275000 & $14.1952 \times 10^{-6}$ & $1233.6421626 \pm 28.5476163$ & -0.981 & 0.965 \\
\hline aaqcozd & 2458257.7778472 & 3 & 716 & 11 & 2 & 2 & 2.695 & 26.275000 & $12.6417 \times 10^{-6}$ & $1212.3373769 \pm 32.0352867$ & -0.981 & 0.965 \\
\hline aaqcozd & 2458257.7787963 & 1 & 716 & 11 & 2 & 2 & 2.643 & 26.275000 & $14.0979 \times 10^{-6}$ & $1151.8020575 \pm 30.9927667$ & -0.981 & 0.965 \\
\hline aaqcozd & 2458257.7797338 & 2 & 716 & 11 & 2 & 2 & 2.541 & 26.275000 & $14.5513 \times 10^{-6}$ & $1182.5142907 \pm 29.5918902$ & -0.981 & 0.965 \\
\hline abdpvnd & 2458364.8118171 & 1 & 646 & 13 & 2 & 2 & 1.916 & 26.106877 & $3.3710 \times 10^{-6}$ & $1082.7538462 \pm 24.8383520$ & -999 & -999 \\
\hline abdpvnd & 2458364.8239120 & 2 & 692 & 1 & 4 & 2 & 2.217 & 25.958831 & $2.8078 \times 10^{-6}$ & $988.7527352 \pm 25.3953545$ & -999 & -999 \\
\hline abdpvnd & 2458364.8460764 & 2 & 692 & 1 & 4 & 2 & 2.296 & 25.948320 & $3.5620 \times 10^{-6}$ & $982.4542010 \pm 27.9368325$ & -999 & -999 \\
\hline abdpvnd & 2458365.8068634 & 2 & 692 & 1 & 4 & 1 & 2.323 & 26.026621 & $6.2705 \times 10^{-6}$ & $307.9495909 \pm 16.4243875$ & -999 & -999 \\
\hline abdpvnd & 2458365.8400579 & 2 & 692 & 1 & 4 & 2 & 2.442 & 26.006744 & $2.9185 \times 10^{-6}$ & $963.6859996 \pm 24.3100430$ & -999 & -999 \\
\hline
\end{tabular}

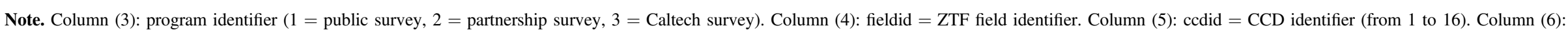

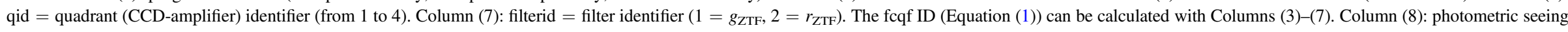

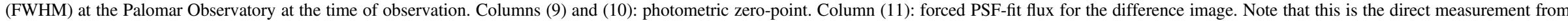

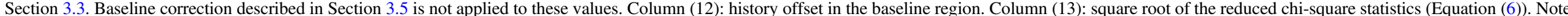

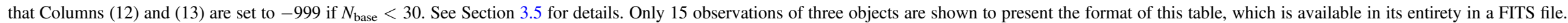




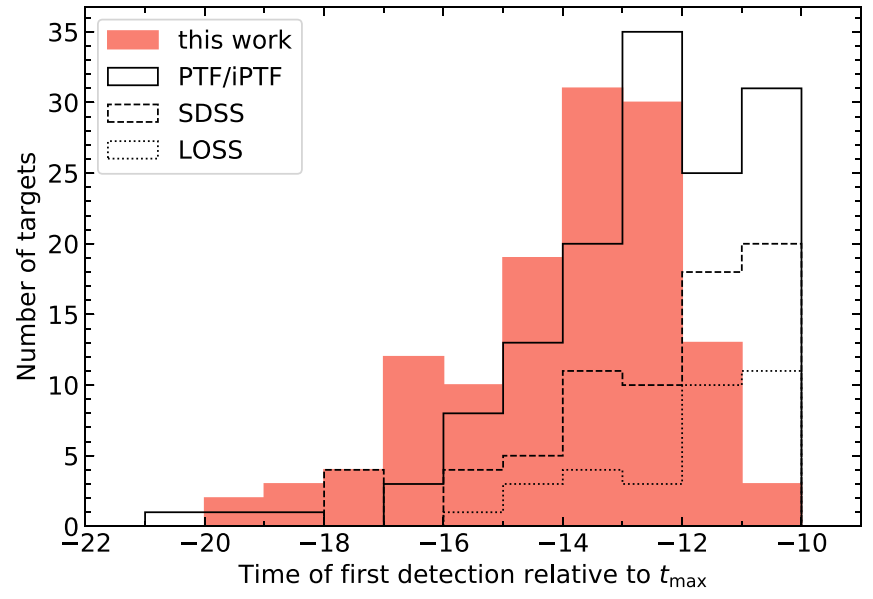

Figure 7. Histogram of first-detection epoch relative to $B$ - or $g$-band maximum light for ZTF, PTF/iPTF, SDSS, and LOSS. Times are given in the rest frame except for the LOSS sample, where we use the observer frame instead. The $x$ axis is truncated at -10 days. For the ZTF sample, the median is -13.6 days and the mean is -13.9 days.

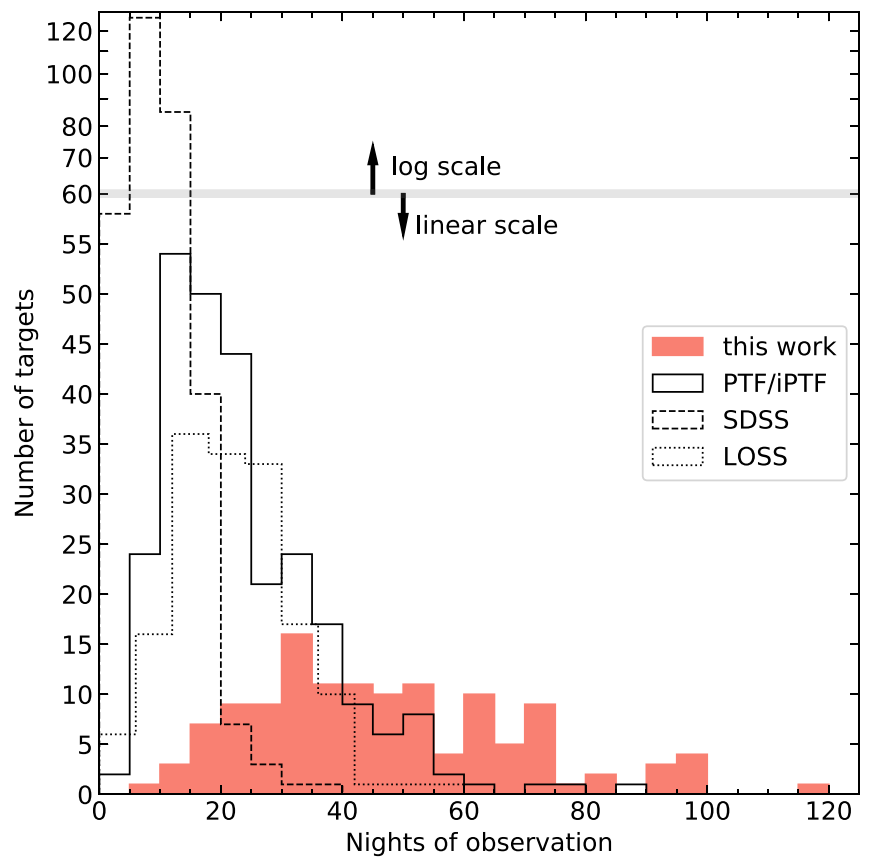

Figure 8. Histogram of the number of nights each SN Ia was observed. For the ZTF sample, the median is 43 nights and the mean is 46 nights.

$M_{g, \text { max }} \gtrsim-19.6$, we tentatively classified them as normal SNe Ia (denoted by "normal*"), and identified those with $M_{g, \max } \lesssim-19.6$ as potentially $91 \mathrm{~T}$-like events (denoted by "91T-like*"). The estimation of absolute magnitude at maximum light can be found in Section 4.3. In Table 2, uncertain subtype determinations are indicated with an asterisk.

If the classification spectra were obtained sufficiently past maximum (spectral phase $>15$ days), we chose to put an asterisk at the end of their SNID classification, since a single late-time spectrum cannot rule out other subtypes. Given that we do not have a spectrum for ZTF18abptsco, we also considered it to be "normal*." As a result, 85 objects were identified as "normal," 26 as "normal*," eight as the "91T-like" subtype, six as "91T-like*," one as "91bg-like," and one as "Ia-CSM."

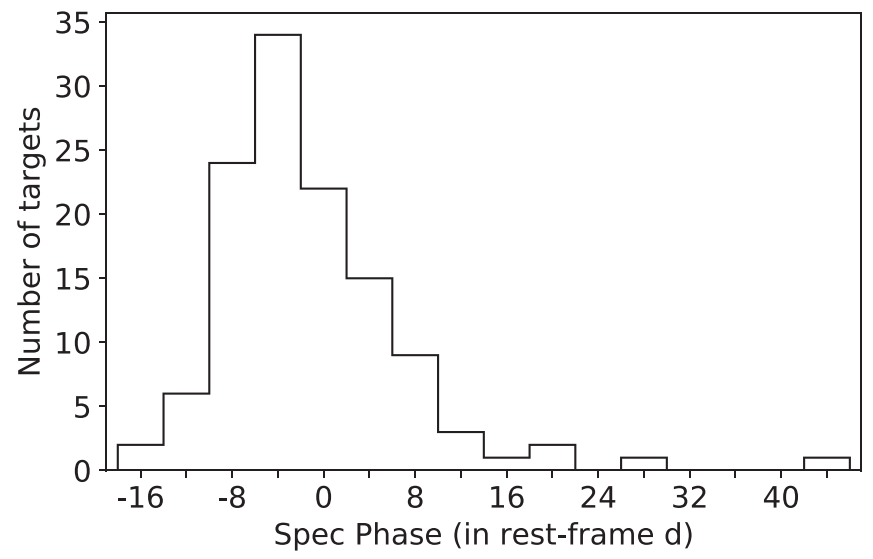

Figure 9. Histogram of the phase of the spectrum with which the classification was made for 120 targets in our sample. The median is -3 days. ZTF18abptsco and the six peculiar objects are not included.

\subsubsection{Subtype Modifications and Reliability}

99aa-like and 91T-like events. SN 1999aa-like events were included in the Ia-91T SNID templates. They are transitional objects between 91T-like and normal SNe Ia, and may represent a spectroscopically distinct subclass (Silverman et al. 2012). Therefore, for targets classified as 91T-like events by SNID, we further inspected their spectra and best matched templates. 99aa-like events were identified via their strong Ca II $\mathrm{H} \& \mathrm{~K}$ lines. Seven objects were classified as 99aa-like after first being 91T-like events, while one object was classified as 99aa-like* after first being 91T-like*.

91bg-like and 86G-like events. The subluminous 86G-like and 02es-like (Ganeshalingam et al. 2012) objects were included in the Ia-91bg SNID templates. For the one object (ZTF18abdmgab) classified by SNID as Ia-91bg, we compared its spectrum at maximum light with that of SN $1991 \mathrm{bg}$, SN 1986G, and SN 2002es. It is clearly more similar to SN 1986G, so we classify it as such.

Objects with multiple spectra. Multiple spectra have been obtained for 31 events in our sample. Among them, 17 got more than one spectrum with a reliable classification. It is worth checking the consistency of the SNID classifications from different spectra, because it will help to answer whether the top three SNID matches having the same subtype means that such a subtype determination is indeed reliable.

We noticed that among the 17 events, SNID subtypes of 15 objects are consistent with each other $(15=13$ "normal" + one "86G-like" + one "99aa-like"). The two exceptions are ZTF18abauprj and ZTF18abclfee. ZTF18abauprj was classified by a NOT spectrum at -6 days as a "99aa-like" event (see Figure 10). This event received an extensive rapid spectroscopic follow-up campaign: a DCT spectrum at -11 days, two APO spectra at -12 and -15 days, a Keck I spectrum at -15 days, as well as an LT spectrum at -15 days. Although typical features of 99aa-like events (Fe III multiplets, strong Ca II H \& K lines, and weak Si II) clearly exist in the five spectra, the SNID classifications, however, are normal SN Ia. The inconsistency may result from the lack of early 91T-like templates in the SNID database, which suggests that the "normal" typing from early-time spectra (phase $<-10$ days) of seven events ${ }^{32}$ in Table 2 may be questionable. However,

\footnotetext{
32 They are ZTF18aazixbw, ZTF18abcflnz, ZTF18abcrxoj, ZTF18abfhryc, ZTF18abkhcrj, ZTF18abkhcwl, ZTF18abmxdhb.
} 
Table 6

Summary of Spectroscopic Observations

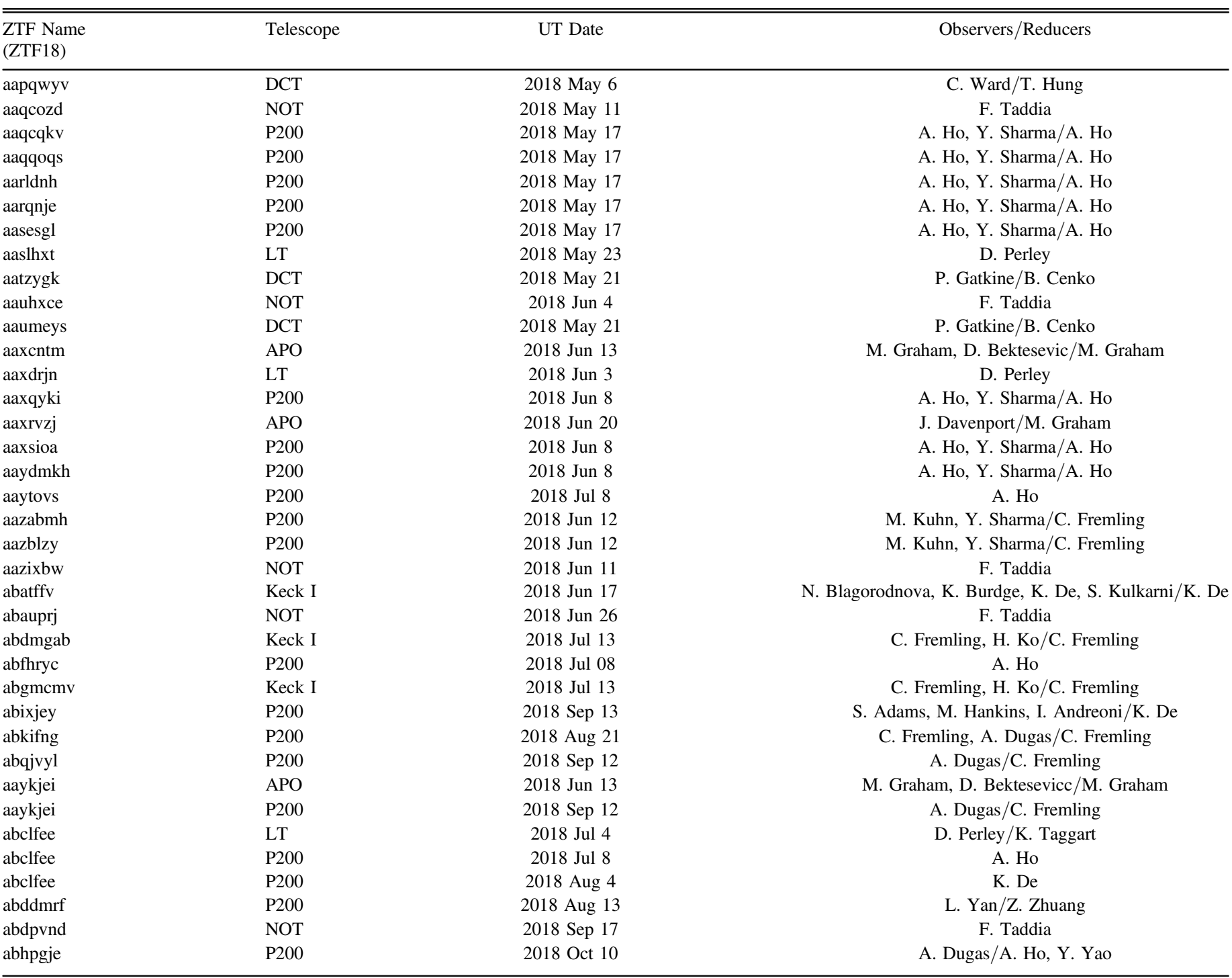

Note. Only non-SEDM spectra are included in this table. SEDM spectra will be described in detail by one of us (M. Rigault et al. 2019, in preparation).

the peak luminosity of these events $\left(M_{g, \max }\right.$ ranges from -18.5 to $-19.2 \mathrm{mag}$ ) is consistent with their being normal SNe.

The fact that SNID has more normal SN Ia templates leads to a subtype "attractor" with the risk for low-S/N spectra to be preferentially classified as normal, regardless of their type. When the number of templates per class is highly unbalanced, then it is far more likely that the statistically best match to a low-S/N spectrum will occur with the dominant class. Classification bias due to the unbalanced training set was noted by Blondin \& Tonry (2007) and has been demonstrated in subsequent studies (e.g., Foley et al. 2009; Ostman et al. 2011; Silverman et al. 2012).

ZTF18abclfee was classified by an SEDM spectrum at +2 days as a normal $\mathrm{SN}$, but later spectra at +4 and +8 days were classified as Ia-pec. This is indeed an 02cx-like event and the classification will be justified in Section 5.3. We also identify four super-Chandrasekhar (SC) mass explosions in Section 5.2. Although they were misclassified as "normal(*)" or "91T-like*" by SNID, they can be distinguished from normal events by their overluminous peak luminosity, lower velocities, and the lack of a distinct second maximum (in the red portion of the optical spectrum) typical of normal $\mathrm{SNe}$ Ia (Scalzo et al. 2010).

Conclusion. Among the $127 \mathrm{SNe}$ Ia in our sample, 82 events were identified as "normal," 25 as "normal*," seven as "99aalike," three as "99aa-like*," one as "91T-like," two as "91Tlike*," one as 86G-like, one as "02cx-like," one as "Ia-CSM," two as "SC," and two as "SC*." The identification of peculiar events will be presented in Section 5. The subtype classification given in Column (8) of Table 2 should be relatively reliable, since we have considered both spectroscopic and photometric properties.

\subsection{Light-curve Properties}

Forced photometry for the $127 \mathrm{SNe}$ Ia in our sample is shown in Figures 11 and 12. We adopted Equations (9) and (10) to convert $f_{\text {ratio }}$ into the observed magnitude. The absolute magnitude is determined by correcting for the distance modulus and Galactic extinction $E(B-V)$ estimated by Schlafly \& 


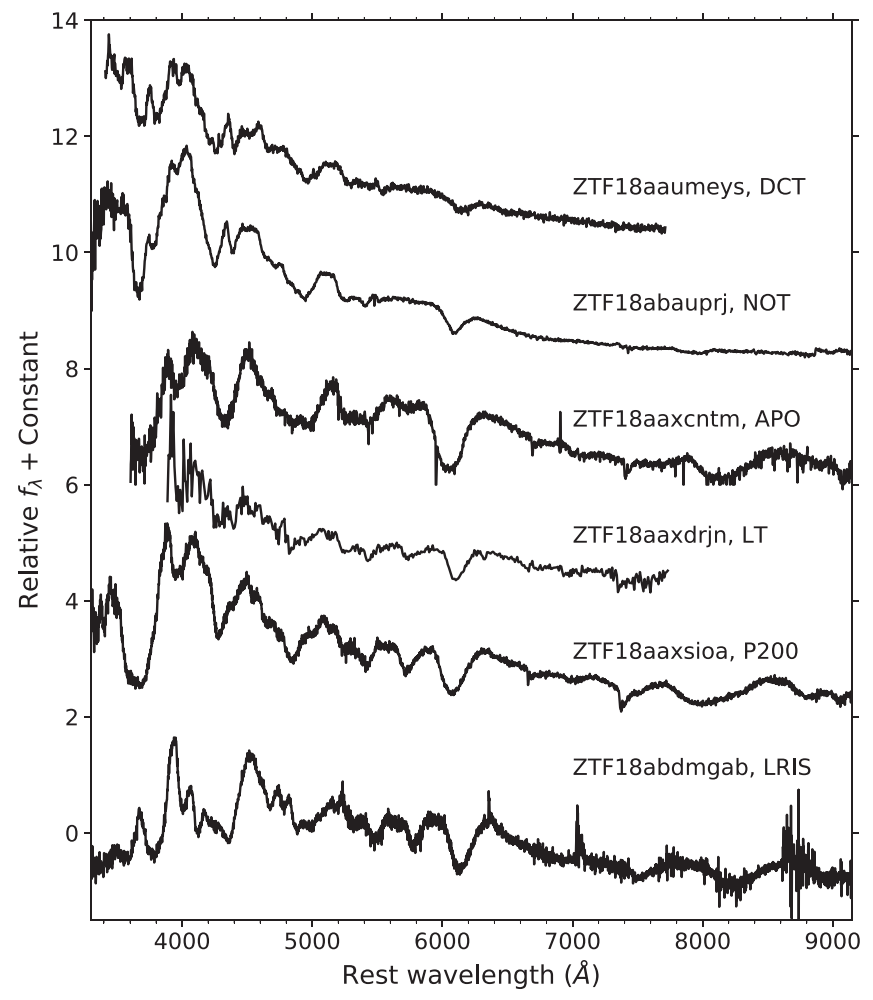

Figure 10. A subset of the spectra used for classification for six objects in our sample. Details of the spectra are given in Table 6.

Finkbeiner (2011), which builds upon Schlegel et al. (1998). We assume $R_{V}=3.1$, and integrate the reddening law from Cardelli et al. (1989) over the ZTF filters.

In order to estimate the time of $g$-band maximum $\left(t_{g, \max }\right)$, observed peak magnitude $\left(m_{g \text {,max }}\right)$, and the decline-rate parameter expressed by the decline within 15 days from maximum in the $g$ band $\left(\Delta m_{15}(g)\right)$, we fit light curves of $\operatorname{normal}(*), 99 \mathrm{aa}-\operatorname{like}(*), 91 \mathrm{~T}-\mathrm{like}(*)$, and $86 \mathrm{G}$-like objects with SALT2 (as shown by the overplotted solid lines in Figure 11). For the 02cx-like, Ia-CSM, and $\mathrm{SC}(*) \mathrm{SNe}$ we fit the light curves with low-order polynomial functions (as shown by the overplotted solid lines in Figure 12). We convert $m_{g \text {,max }}$ into absolute peak magnitude $M_{g, \max }$ using the same method as above.

We separate the sample into two groups because SALT2 is currently not suitable to satisfactorily determine light-curve features of peculiar events. Our choice of low-order polynomial functions follows the fitting technique adopted by Foley et al. (2013) and Miller et al. (2017). Estimated parameters are reported in Table 3.

\subsubsection{Light-curve Fitting with SALT2}

To attempt the SALT2 fits, we assumed $R_{V}=3.1$, adopted the Galactic extinction estimate of $E(B-V)$ from Schlafly \& Finkbeiner (2011), and added a Milky Way dust model (CCM89Dust) (Cardelli et al. 1989) to the SN model (SALT2Model) in sncosmo (Barbary et al. 2016). SALT2 characterizes the flux density for a given $\mathrm{SN}$ as a function of phase $p$ and rest-frame wavelength $\lambda$ as

$$
f(p, \lambda)=x_{0}\left[\mathcal{M}_{0}(p, \lambda)+x_{1} \mathcal{M}_{1}(p, \lambda)\right] e^{c \cdot \mathcal{C}_{\mathrm{L}}(\lambda)}
$$

where $x_{0}, x_{1}$, and $c$ are the normalization, shape, and color parameters, respectively. The mean spectral sequence $\mathcal{M}_{0}$, the first-order deviation around the mean sequence $\mathcal{M}_{1}$, and the average color-correction law $\mathcal{C}_{\mathrm{L}}$ were trained on photometric and spectroscopic data of known SNe Ia (see Guy et al. 2007 for details).

In addition to $t_{g, \max }, M_{g, \max }$, and $\Delta m_{15}(g)$, we also obtained the expected $\Delta m_{15}(B)$ and $M_{B, \max }$ from the SALT2 fitted parameters for objects shown in Figure 11.

\subsubsection{Light-curve Fitting with Polynomial Functions}

We interpolated the observed photometry of peculiar events with low-order polynomial fits. The degree of the polynomial used to fit the light curve is chosen between 2 and 3, optimized using the Bayesian information criteria (BIC; Schwarz 1978). According to BIC, another parameter should only be added (by increasing the order of the polynomial) if it decreases $\chi^{2}$ by at least the natural logarithm of the number of data points. For the five overluminous events (one Ia-CSM and four SC(*)), the time range used in the fit is from -10 to +16 days (in the rest frame) relative to maximum light. For the $02 \mathrm{cx}$-like object we only fit data points from -5 to +16 days (in the rest frame) relative to maximum light.

We obtained the covariance matrix of the polynomial coefficients (Cov) with numpy's polyfit function. To estimate uncertainties of $t_{g, \max }, M_{g, \max }$, and $\Delta m_{15}(g), 100,000$ Monte Carlo simulations were run by resampling the polynomial coefficients from the Cholesky decomposition of Cov.

\subsubsection{The Selection Effect}

Figure 13 shows the distribution of $127 \mathrm{SNe}$ in the plane of the light-curve shape parameter SALT2 $x_{1}$ versus redshift. Larger $x_{1}$ indicates a broader light curve and greater maximum luminosity. We note that the correlation between $x_{1}$ and $\Delta m_{15}(g)$ is sufficiently strong that $x_{1}$ can be used as a proxy for the rate of decline of the light curve and thus the peak luminosity (see Figure 15). The median of $x_{1}$ should be around zero for an unbiased sample. For our sample, $x_{1}$ is centered at $\sim 0$ for 100 SNe with $z \leqslant 0.1$ (median $x_{1}=0.007$ ), but shifts to greater values for the 27 objects with $z>0.1$ (median $\left.x_{1}=0.729\right)$. This is a consequence of Malmquist bias (Malmquist 1922) — at higher redshift, only targets intrinsically more luminous can be detected early enough. The Malmquist bias had been predicted at about the same level from the PTF/ iPTF work (see Figure 11 of Papadogiannakis et al. 2019).

\subsubsection{The Luminosity-Decline Relation}

Accurate estimates of host extinction are critical for the luminosity-decline relation of SNe Ia (Phillips 1993). An empirical way to get the absolute $B$-band magnitude corrected for host extinction is to subtract 3.1 times the SALT2 color parameter $(c)$ from $M_{B, \max }$ corrected for Galactic extinction (Betoule et al. 2014). The $c$-corrected $M_{B, \max }$ is plotted against the rate of decline of the light curve $\Delta m_{15}(B)$ for $121 \mathrm{SNe}$ in Figure 14. Peculiar events shown in Figure 12 are not included since their light curves cannot be well fitted by SALT2. There is a fairly tight correlation between luminosity and decline rate for normal SNe Ia plotted as filled black circles. 

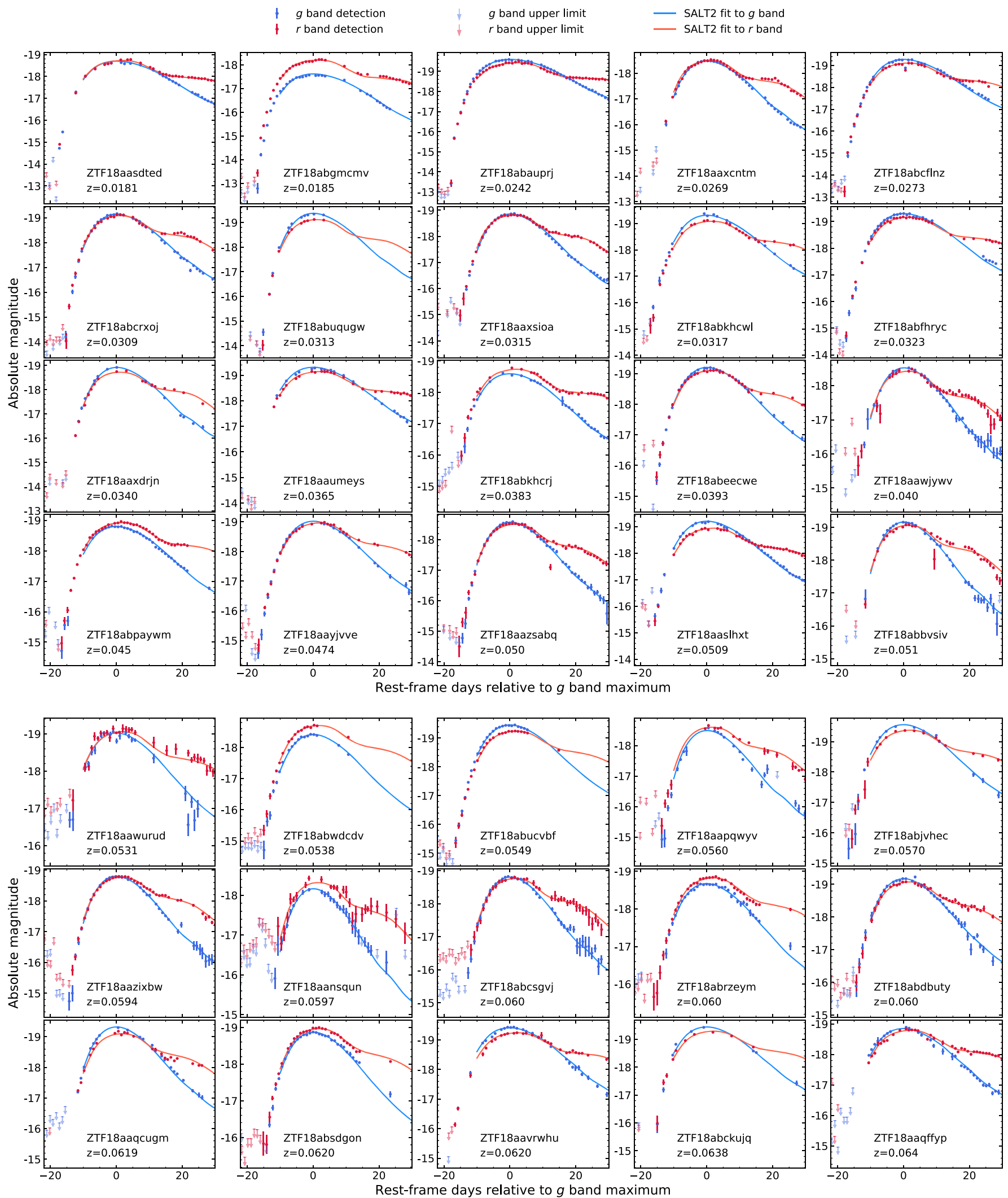

Figure 11. P48 light curves of $121 \mathrm{SNe}$ Ia classified as normal $(*)$, 91T-like $(*)$, 99aa-like $(*)$, and 86G-like in our sample. Blue and crimson data points show detections in the $g$ and $r$ bands, respectively. $5 \sigma$ upper limits are shown with downward-pointing arrows. Solid lines are SALT2 fits to the data. Absolute magnitude has been corrected for Galactic extinction (host extinction is assumed to be zero for all targets). Single-night observations in the same filter are binned (by taking the inverse variance-weighted average) for illustration. Targets are ordered by redshift. 

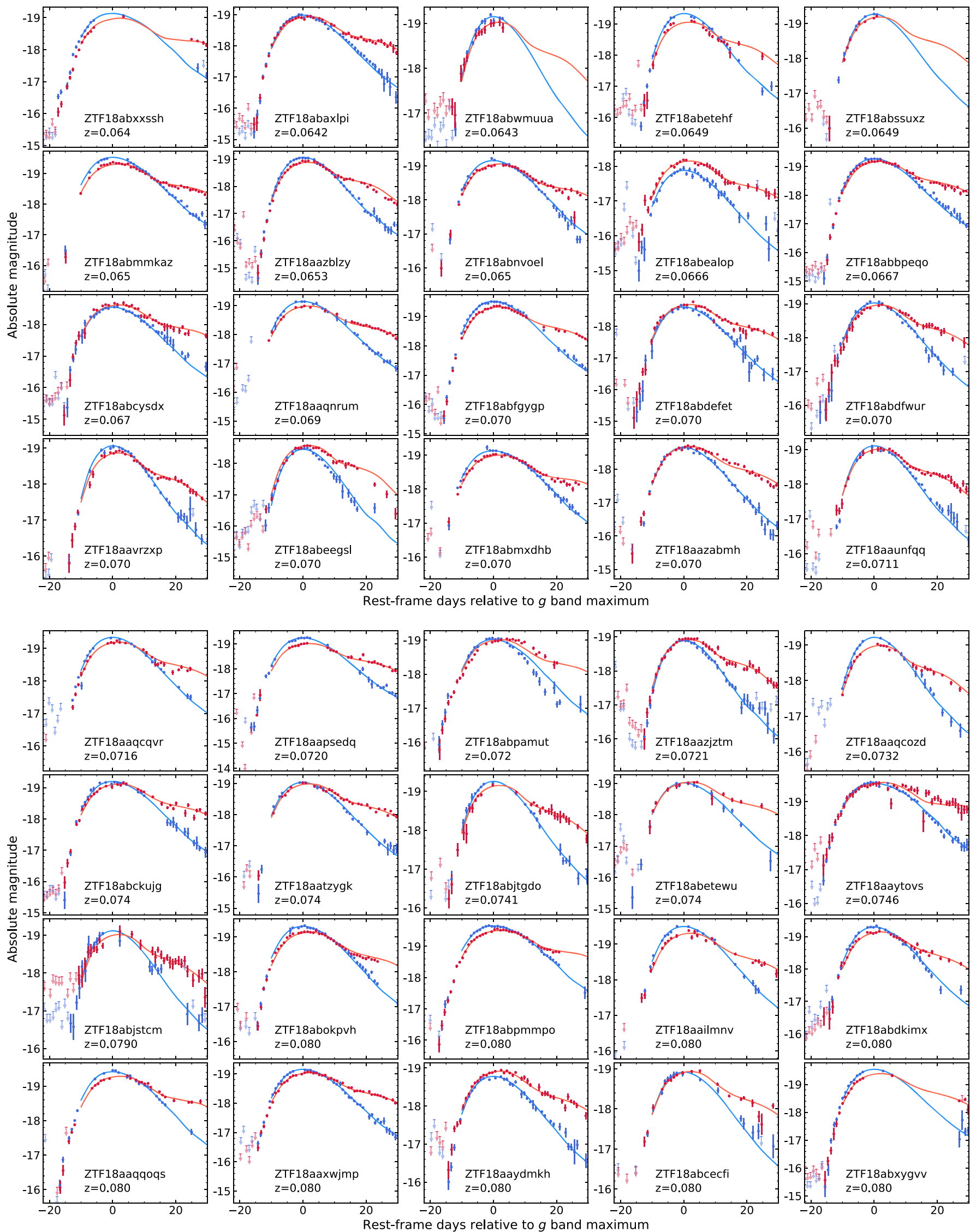

Figure 11. (Continued.) 

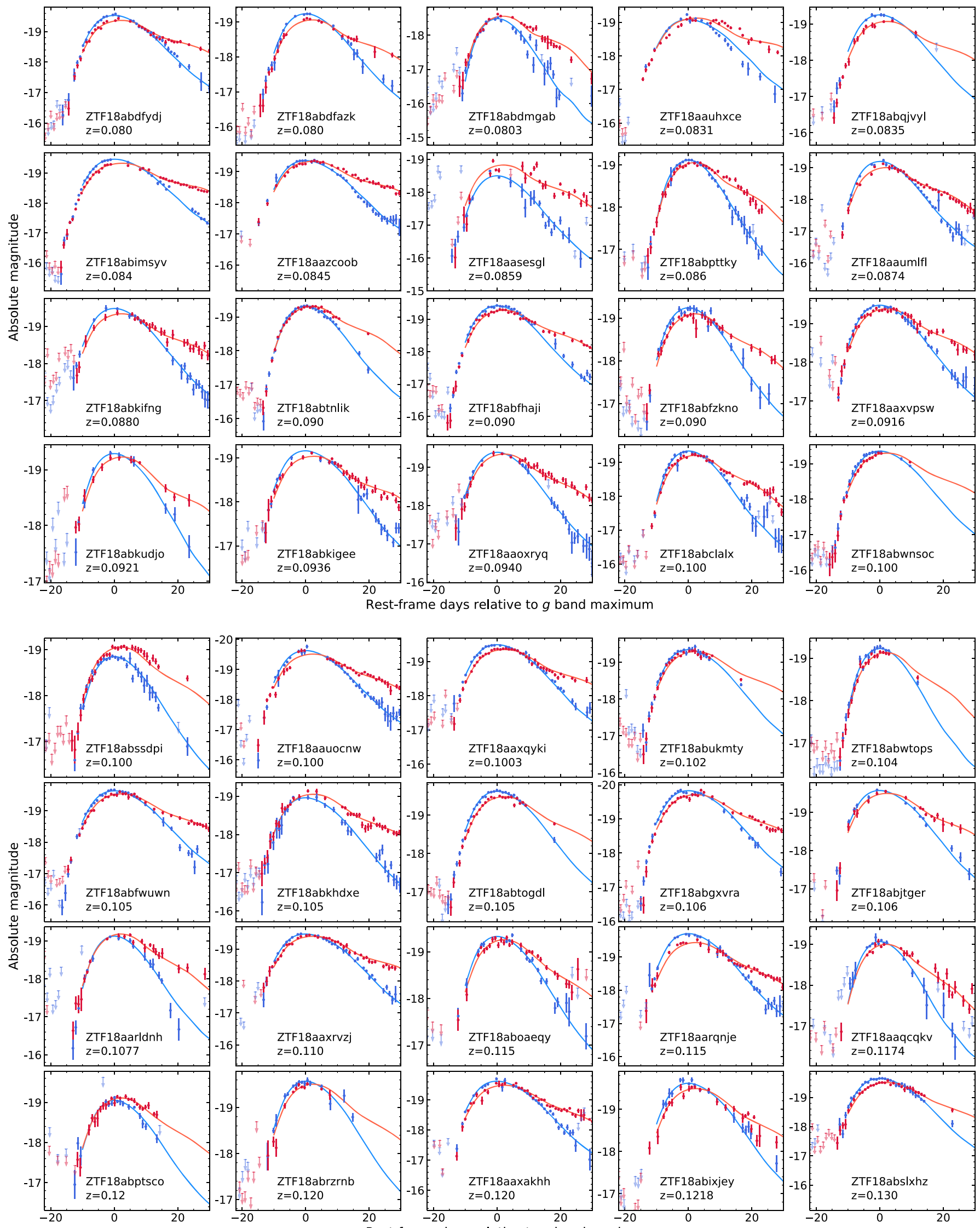

Figure 11. (Continued.) 


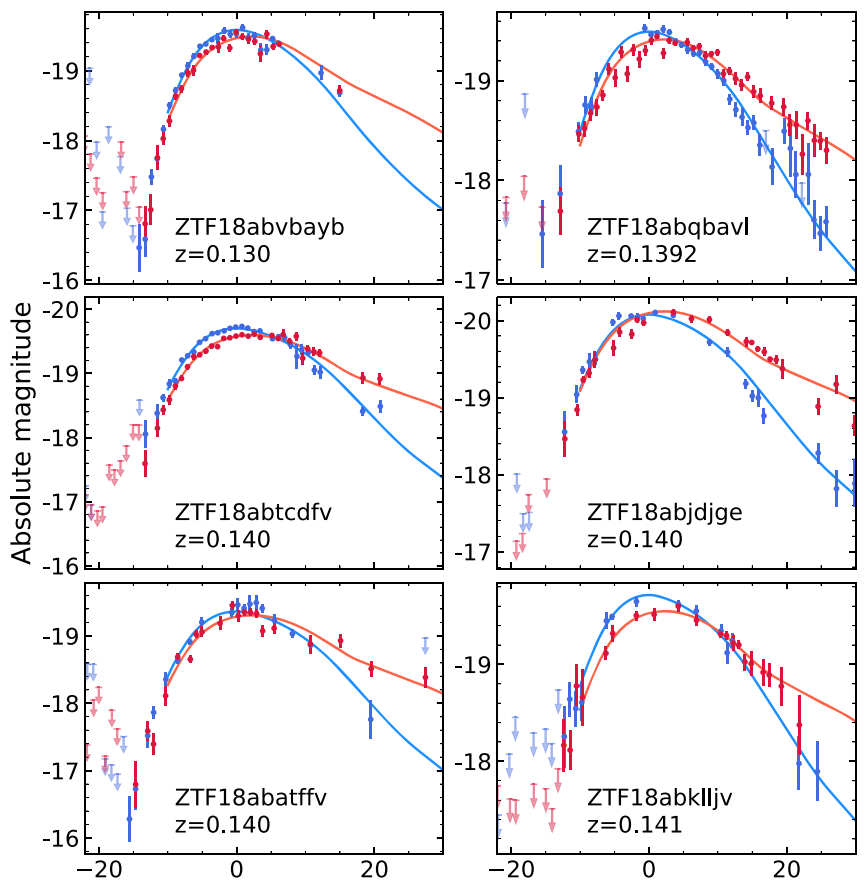

Figure 11. (Continued.)

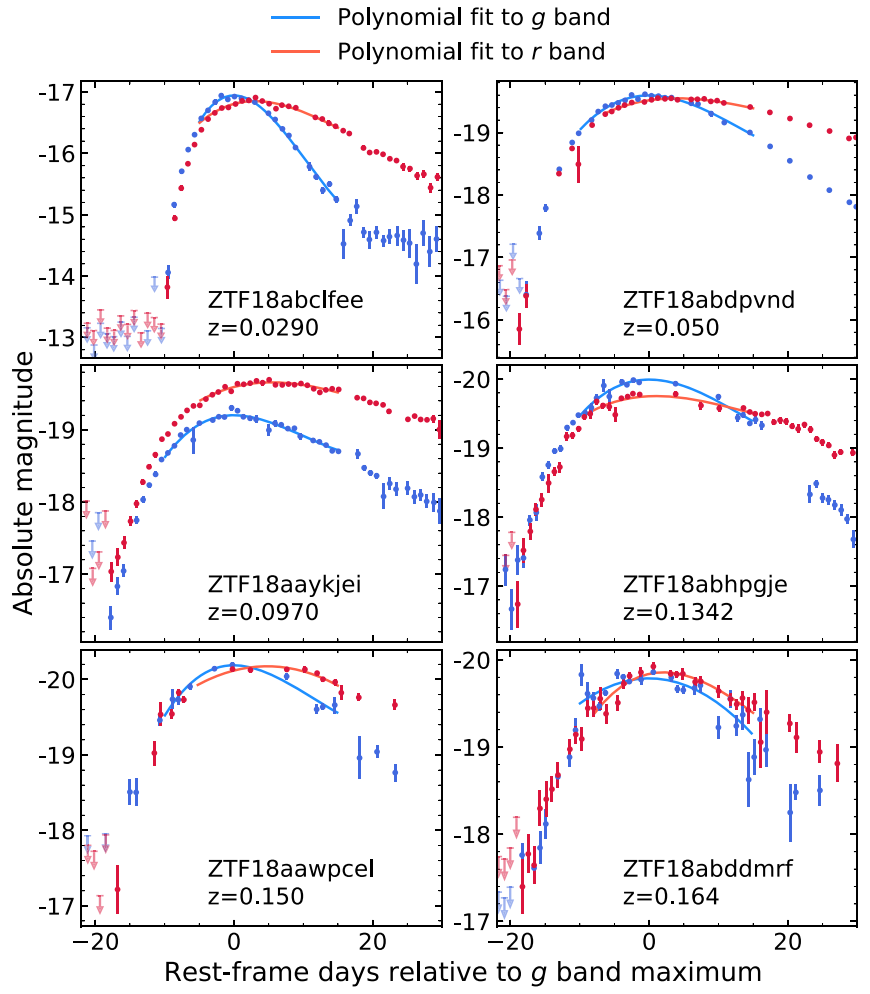

Figure 12. P48 light curves of six peculiar SNe Ia in our sample. Solid lines are polynomial fits to the data. See Figure 11 for symbols.

\section{Peculiar Events}

It has been demonstrated that for peculiar events, in the absence of a constraint on redshift, the fraction of correct subtype matches is low due to the limited number of peculiar templates in the database (Blondin \& Tonry 2007). However, peculiar events can also be selected by their unusual light-curve

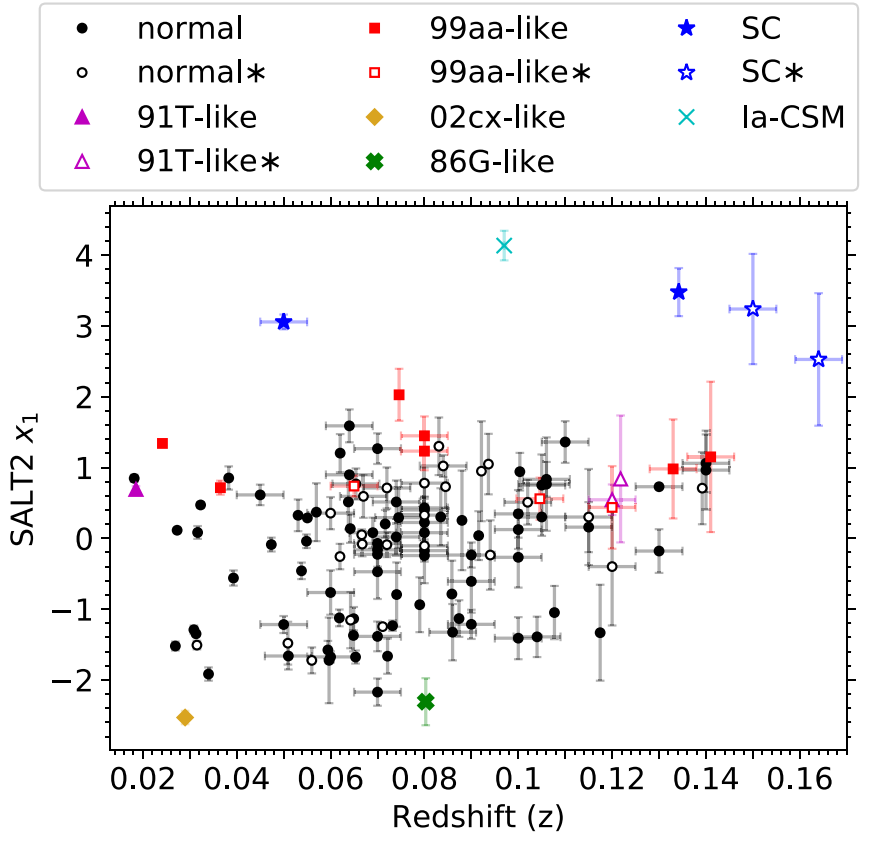

Figure 13. SALT2 $x_{1}$ vs. redshift $(z)$ for the $127 \mathrm{SNe}$ in our sample. The apparent positive correlation between $x_{1}$ and redshift is due to the Malmquist bias (fainter events, i.e., low $x_{1}$, are not detected at $z \gtrsim 0.1$ ). Section 5 discusses the peculiar events.

properties. Figure 15 shows the distribution of $127 \mathrm{SNe}$ Ia in the plane of SALT2 $x_{1}$ versus decline rate. The light-curve shape parameter $x_{1}$ closely tracks $\Delta m_{15}(g)$ within uncertainties for normal, 99aa-like, 91T-like, and 86G-like $\mathrm{SNe}$ in our sample. A linear fit gives

$$
x_{1}=(-9.13 \pm 0.09) \times \Delta m_{15}(g)+(7.43 \pm 0.06) .
$$

There are six events with $x_{1}>2$. Except for ZTF18aaytovs, which is spectroscopically classified as a 91T-like object 


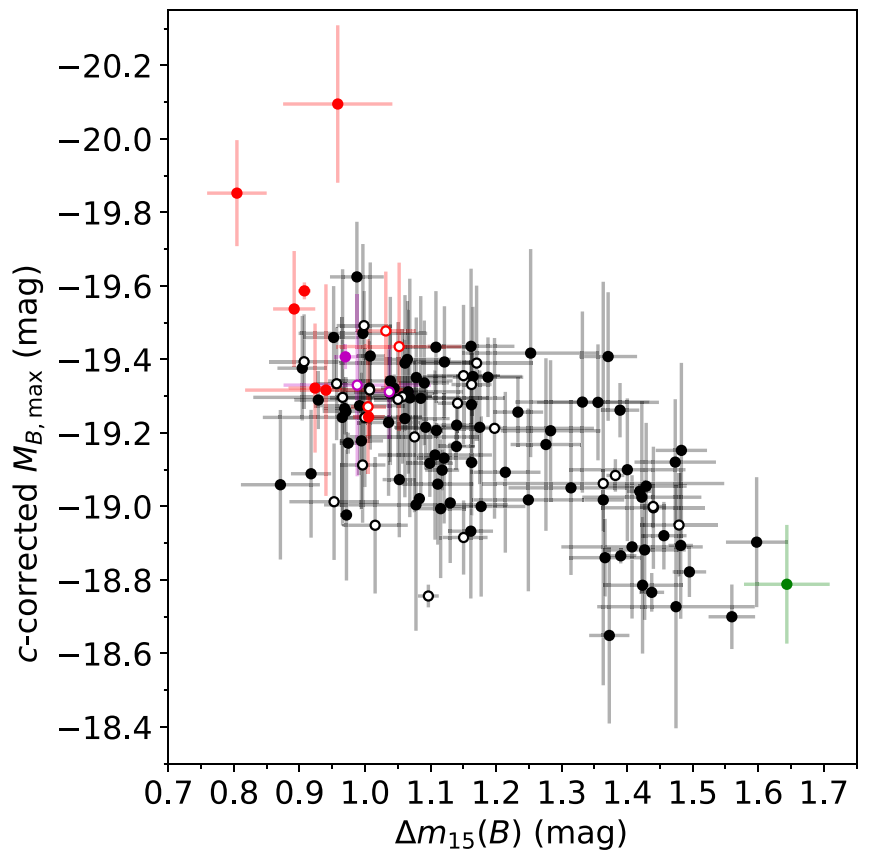

Figure 14. Distribution of $121 \mathrm{SNe}$ in the plane of SALT2 $c$-corrected $M_{B \text {, max }}$ vs. $\Delta m_{15}(B)$. Symbol colors follow the same convention as in Figure 13.

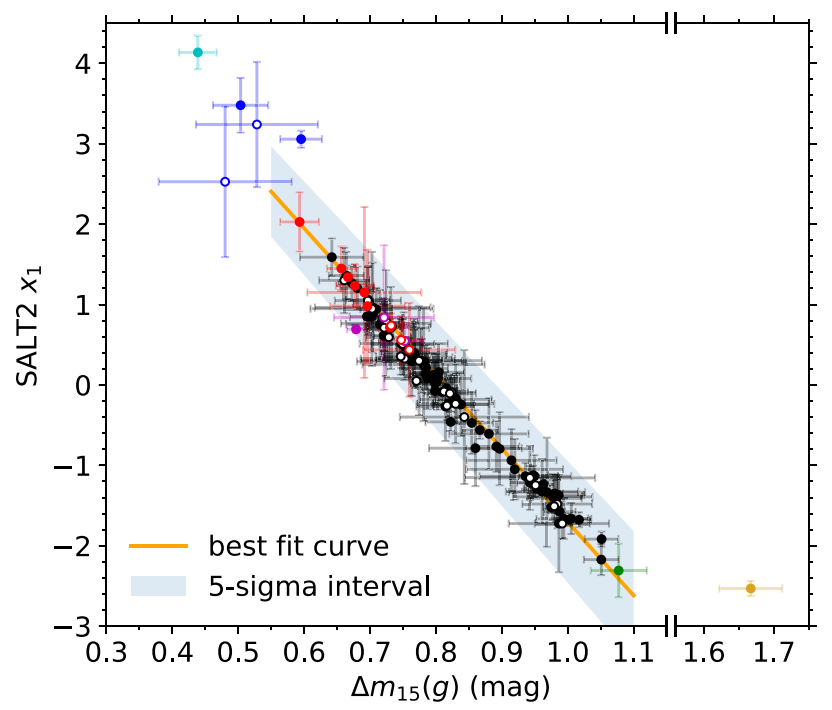

Figure 15. Distribution of $127 \mathrm{SNe}$ in the plane of SALT2 $x_{1}$ vs. $\Delta m_{15}(g)$. Symbol colors follow the same convention as in Figure 13. The solid line shows a linear fit to non-peculiar objects (data shown in black, red, magenta, and green). Note the broken axis.

(Section 4.2), the other five objects are all peculiar overluminous slow decliners, and are discussed below. The one object shown in yellow (ZTF18abclfee) has the smallest value of $x_{1}$, and declines much faster than other $\mathrm{SNe}$ (note the broken $x$-axis). These events can all be classified as "peculiar," below we illustrate their observational characteristics to justify their classification.

\subsection{A Ia-CSM SN: ZTF18aaykjei (SN 2018crl)}

Ia-CSM is a subclass of $\mathrm{SNe}$ Ia showing evidence of interaction between the ejecta and the dense circumstellar medium (Dilday et al. 2012). As can be seen in Figure 16, the spectra of ZTF18aaykjei are dominated by $\mathrm{H} \alpha$ emission at all

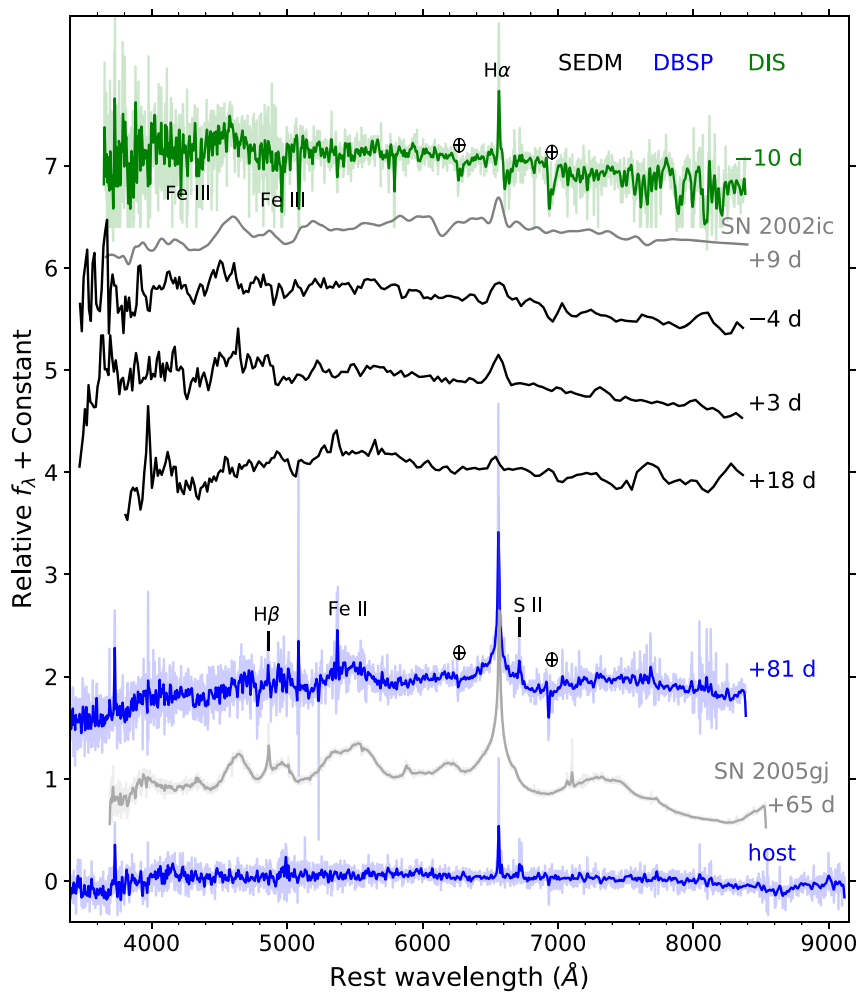

Figure 16. Spectra of ZTF18aaykjei in comparison to two other Ia-CSM objects (SN 2002ic and SN 2006gj). The original APO and DBSP spectra are shown in translucent colors, with the overlying solid lines showing the same spectra convolved with a Gaussian kernel of $\mathrm{FWHM}=500 \mathrm{~km} \mathrm{~s}^{-1}$. The spectrum of its host galaxy is shown at the bottom.

epochs. Its early-time APO spectrum matches the prototype of Ia-CSM SNe (SN 2002ic), whose spectral features resembled 1991T-like events, but diluted in strength (Hamuy et al. 2003). Absorption and emission lines of intermediate-mass elements and iron-peak elements (IPEs) are present in the blue portion of the two spectra at -4 and +3 days, but the $\mathrm{Si}$ II lines commonly seen in normal SNe Ia spectra are "veiled" by continuum radiation. The noisy SEDM spectrum at +18 days only matches to an Sb-type galaxy spectrum in the SNID database. Its late-time DBSP spectrum matches the Ia-CSM object SN 2005gj (Aldering et al. 2006), where emission lines from overlapping IPEs (mostly Fe II) are prominent.

The redshift $(z=0.0970)$ of ZTF18aaykjei is measured from the $\mathrm{H} \alpha,[\mathrm{N}$ II] $\lambda \lambda 6548,6583$, and [S II] $\lambda \lambda 6716,6731$ (nebular) lines in the spectrum of its host galaxy (SDSS J161938.91 +491104.7) obtained by DBSP on 2019 May 24. At +81 days, the $\mathrm{H} \alpha$ emission line profiles have a narrow component on top of a broad $\left(\mathrm{FWHM} \approx 1020 \mathrm{~km} \mathrm{~s}^{-1}\right.$ ) base, much greater than the $\mathrm{H} \alpha$ FWHM of the host-only spectrum $\left(97 \mathrm{~km} \mathrm{~s}^{-1}\right)$. The spectra presented here have relatively low resolution, so we do not expect to resolve the P-Cygni profiles seen in some other Ia-CSM SNe.

We show the light curve of ZTF18aaykjei in the middle left panel of Figure 12. It peaked at $-19.19 \pm 0.04 \mathrm{mag}$ in $g$ and $-19.65 \pm 0.04 \mathrm{mag}$ in $r$ (only Galactic extinction is corrected in these estimates). Its red color even at early times suggests that the host extinction may be non-negligible. The peak luminosity of ZTF18aaykjei is consistent with other objects in the Ia-CSM class $\left(-21.3 \mathrm{mag} \leqslant M_{R} \leqslant-19 \mathrm{mag}\right.$, Silverman et al. 2013). 

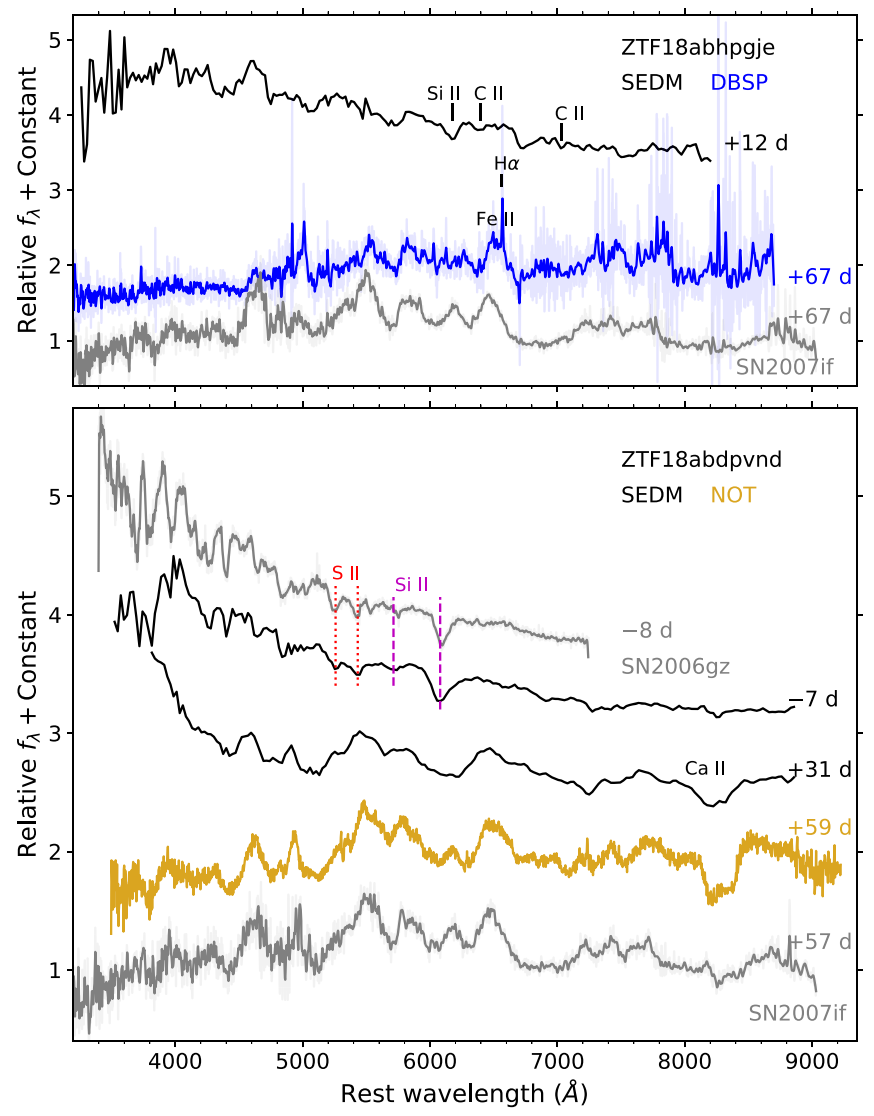

Figure 17. Upper panel: spectra of ZTF18abhpgje. Si II and C II lines at $8000 \mathrm{~km} \mathrm{~s}^{-1}$ are marked. Bottom panel: spectra of ZTF18abdpvnd. Si II features at $13,000 \mathrm{~km} \mathrm{~s}^{-1}$ and $\mathrm{S}$ II features at $10,000 \mathrm{~km} \mathrm{~s}^{-1}$ are marked. Spectra of SN 2009gz and SN 2007if are obtained from WISEReP and are shown in gray. The DBSP spectrum is smoothed by a Gaussian kernel with $\mathrm{FWHM}=500 \mathrm{~km} \mathrm{~s}^{-1}$.

\subsection{Super-Chandrasekhar Explosions}

There are four events in our sample with $x_{1} \approx 3$ : ZTF18abhpgje, ZTF18abdpvnd, ZTF18aawpcel, and ZTF18abddmrf. Their light curves are displayed in Figure 12. Among them, the former two events are considered to be super-Chandrasekhar mass explosions (termed "SC" in Table 2) with evidence from spectroscopy, while the latter two events are classified as candidate super-Chandrasekhar SNe (denoted as "SC*" in Table 2) based only on their light curves.

\subsubsection{ZTF18abhpgje (SN 2018eul)}

The redshift of ZTF18abhpgje was measured to be 0.134 from the host $\mathrm{H} \alpha$ emission in its DBSP SN spectrum (upper panel of Figure 17). After correcting for Galactic extinction, it peaked at $-19.99 \pm 0.04 \mathrm{mag}$ in $g$ and at $-19.74 \pm 0.03 \mathrm{mag}$ in $r$. Si II $\lambda 6355$ and C II $\lambda \lambda 6580,7234$ absorption features can be identified in its SEDM spectrum, with velocities of $\sim 8000 \mathrm{~km} \mathrm{~s}^{-1}$. The measured velocity is slower than normal SNe Ia $\left(\sim 10,000 \mathrm{~km} \mathrm{~s}^{-1}\right)$ but similar to some other superChandrasekhar mass SN candidates: at +12 days, SN 2007if has $v($ Si II $\lambda 6355) \sim 8600 \mathrm{~km} \mathrm{~s}^{-1}$ (Scalzo et al. 2010) and SN 2009dc has $v$ (Si II $\lambda 6355) \sim 7500 \mathrm{~km} \mathrm{~s}^{-1}$ (Yamanaka et al. 2009). The extreme luminosity and low velocity have been interpreted as the result of either high gravitational binding energy (Howell et al. 2006) or the deceleration of the outer layers of ejecta by a massive envelope surrounding the progenitor (Scalzo et al. 2010). The best match to its latetime DBSP spectrum is SN 2017if at +67 days (also shown in the upper panel of Figure 17 for comparison), which further supports the argument that ZTF18abhpgje has a superChandrasekhar mass progenitor.

\subsubsection{ZTF18abdpvnd (SN 2018dvf)}

The redshift of ZTF18abdpvnd was inferred to be 0.05 by SNID. After accounting for Galactic extinction, it peaked in $g$ and $r$ bands at $-19.59 \pm 0.26 \mathrm{mag}$ and $-19.56 \pm 0.23 \mathrm{mag}$, respectively. ${ }^{33}$ This is slightly fainter than other overluminous peculiar objects in our sample, but is still significantly more luminous than normal SNe Ia. As can be seen in the bottom panel of Figure 17, at -7 days, intermediate-mass elements ( $\mathrm{Si}$ II $\lambda \lambda 5872,6355$ at $\sim 13,000 \mathrm{~km} \mathrm{~s}^{-1}, \mathrm{~S}$ II at $\sim 10,000 \mathrm{~km} \mathrm{~s}^{-1}$ ) are clearly present in its SEDM spectrum. This is similar to the early-time velocity of SN 2006gz (Hicken et al. 2007), which is another well-observed SN with a super-Chandrasekhar mass progenitor. There are other well-matched spectral features between ZTF18abdpvnd and SN 2006gz. At +31 days, although the blue side of ZTF18abdpvnd's SEDM spectrum may be affected by calibration issues, $\mathrm{Ca}$ II can be detected on the red side. The best match to its late-time NOT spectrum is SN 2017 if at +57 days.

\subsubsection{ZTF18aawpcel (SN 2018cir) and ZTF18abddmrf (SN 2018dsx)}

The redshifts of ZTF18aawpcel and ZTF18abddmrf were inferred to be 0.150 and 0.164 by SNID, respectively. We note that at such high redshifts $\sigma_{z} \gtrsim 0.01$. The +0 days SEDM spectrum of ZTF18aawpcel shows $\mathrm{Si}$ II at $\sim(14,000 \pm$ $3000) \mathrm{km} \mathrm{s}^{-1}$, which is consistent with the typical peak-time velocity of some normal SNe Ia (Blondin et al. 2012). The +37 days SEDM spectrum of ZTF18abddmrf also matches normal SNe Ia templates. However, their extreme luminosity (ZTF18aawpcel: $\quad M_{g, \text { max }}=-19.95 \pm 0.11 \mathrm{mag}, \quad M_{r, \text { max }}=$ $-19.91 \pm 0.11 \mathrm{mag} ; \quad$ ZTF18abddmrf: $M_{g, \max }=-19.79 \pm$ $0.11 \mathrm{mag}, M_{r, \max }=-19.85 \pm 0.08 \mathrm{mag}$ ) and slow rates of decline make them good candidates for super-Chandrasekhar mass explosions.

\subsection{An 02cx-like Event: ZTF18abclfee (SN 2018cxk)}

ZTF18abclfee is an 02cx-like event at $z=0.029$ (redshift measured from host $\mathrm{H} \alpha$ emission in its +35 days DBSP spectrum). This subclass is also termed "Type Iax supernovae" (SNe Iax) (Foley et al. 2013). Its photometric and spectroscopic properties are concordant with the criteria of this subclass (Foley et al. 2013; White et al. 2015): (1) there is no evidence of hydrogen in any spectra; (2) the velocity of Si II $\lambda 6355$ in the +2 days SEDM spectrum is $\sim 6000 \mathrm{~km} \mathrm{~s}^{-1}$, which is much slower than normal SNe Ia; (3) it shows spectral similarity with other 02 cx-like events, as can be seen in Figure 18; (4) it is a fast-declining, low-luminosity event.

We do not detect narrow Na I D at the redshift of 0.029 in any of our spectra, and therefore we assume that host-galaxy extinction is negligible. This assumption is supported by the observed blue color of ZTF18abclfee at peak (upper left panel of Figure 12). After correcting for foreground Galactic extinction, we find that ZTF18abclfee peaked at $M_{g, \max }=-16.93 \pm 0.06 \mathrm{mag}$,

\footnotetext{
33 Note that the reported uncertainties of peak magnitudes also take the uncertainty of redshift into consideration, and thus are relatively large.
} 


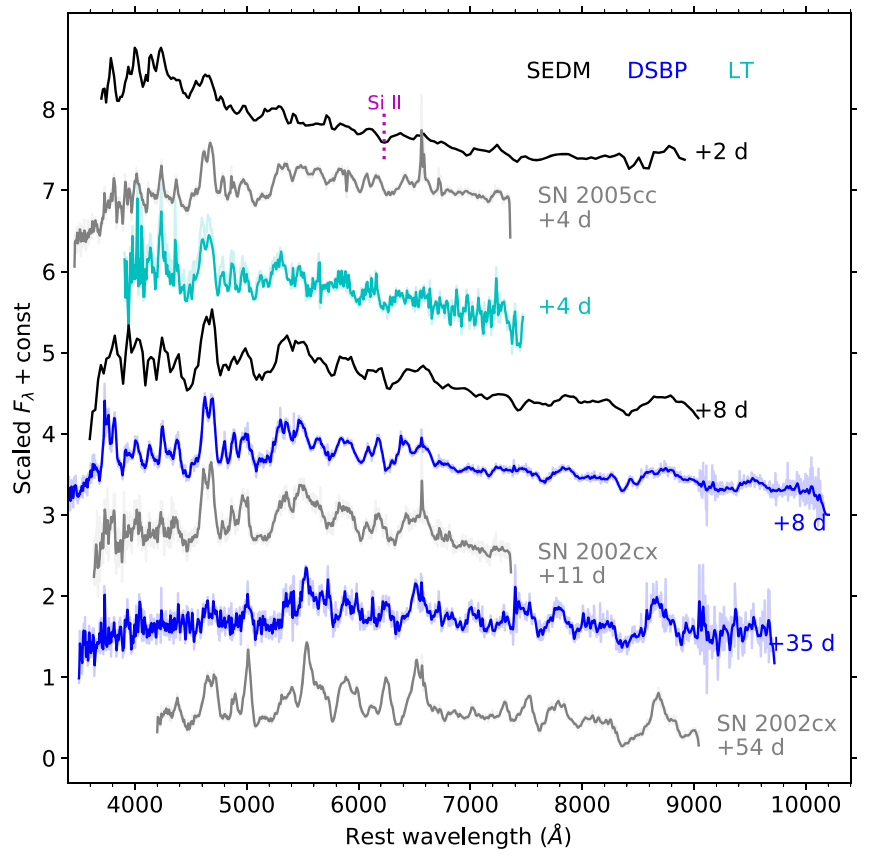

Figure 18. Comparison of spectra of ZTF18abclfee to SN 2002cx and SN $2005 \mathrm{cc}$. The magenta dotted line indicates Si II $\lambda 6355$ at a velocity of 6000 $\mathrm{km} \mathrm{s}^{-1}$. Non-SEDM spectra are smoothed by a Gaussian filter with $\mathrm{FWHM}=500 \mathrm{~km} \mathrm{~s}^{-1}$.

$M_{r, \max }=-16.85 \pm 0.02 \mathrm{mag}$. The decline rates inferred from polynomial fits are $\Delta m_{15}(g)=1.76 \pm 0.08 \mathrm{mag}$ and $\Delta m_{15}(r)=$ $0.56 \pm 0.02 \mathrm{mag}$, which are consistent with other 02cx-like objects (see Figure 8 of Miller et al. 2017). The color evolution of ZTF18abclfee is also consistent with the relations shown in Figure 9 of Miller et al. (2017): at maximum light this event has $(g-r)_{\max } \approx-0.1 \mathrm{mag}$, which is bluer than 91bg-like events and similar to normal SNe Ia; $\Delta(g-r)_{10}=$ $(g-r)_{+10 \text { days }}-(g-r)_{\max } \approx 0.8 \mathrm{mag}$, which is similar to 91bg-like events but much greater than $\Delta(g-r)_{10}$ of normal SNe Ia.

\section{Summary}

In this paper, we have presented an initial data release for ZTF-discovered SNe Ia with early high-cadence observations. The sample covers 2018 (May-December), and features 127 $\mathrm{SNe}$ with dense photometric coverage and early detections in both the $g$ and $r$ bands, allowing an investigation of the initial rise and color evolution. Hence, this sample is well suited for probing the properties of the progenitors of SNe Ia.

By comparing our sample with existing samples of $\mathrm{SNe}$ Ia with low to intermediate redshift, we have demonstrated that our sample stands alone in terms of size and early detections. We developed a custom forced-PSF photometry pipeline to extract high-quality light curves; these methods can also be applied to other types of extragalactic transients. ${ }^{34}$

All of the $127 \mathrm{SNe}$ have forced-photometry detections earlier than 10 days prior to $g$-band maximum light (in the rest frame). Their redshifts range from $z=0.01815$ to $z=0.165$, with a median $z=0.074$. The fact that at $z \gtrsim 0.1$, the majority of SNe $(22 / 27)$ have positive values of the light-curve shape parameter $\left(x_{1}\right)$ suggests that our sample is biased toward overluminous,

\footnotetext{
34 A Python implementation of this method is available at https://github. com/yaoyuhan/ForcePhotZTF.
}

slowly declining SNe at higher redshift. On average, each SN in our sample has been detected in observations on 46 separate nights.

Although detailed spectroscopic examination is beyond the scope of this paper, we present the spectral sequence of four peculiar events in our sample: one Ia-CSM event ZTF18aaykjei (SN 2018crl), one 02cx-like event ZTF18abclfee (SN 2018cxk), two objects with possible super-Chandrasekhar mass progenitors ZTF18abhpgje (AT 2018eul) and ZTF18abdpvnd (SN 2018dvf). Furthermore, ZTF18aawpcel (SN 2018cir) and ZTF18abddmrf (AT 2018dsx) also exhibit photometric properties that are similar to other super-Chandrasekhar mass explosions.

Yuhan Yao thanks Yuping Huang for useful discussions on implementing forced photometry, and the Heising-Simons Foundation for financial support. We gratefully thank Yashvi Sharma, Zhuyun Zhuang, Dino Bektešević Charlotte Ward, Scott Adams, and Igor Andreoni for help with observations. The authors acknowledge Michael Fausnaugh and Seméli Papadogiannakis for sharing data from the TESS and PTF/ iPTF samples, respectively, and an anonymous referee for useful comments that improved the paper.

This work was supported by the GROWTH project funded by the National Science Foundation under PIRE grant No. 1545949. The data presented here were obtained [in part] with ALFOSC, which is provided by the Instituto de Astrofisica de Andalucia (IAA) under a joint agreement with the University of Copenhagen and NOTSA. M.B. acknowledges support from the Swedish Research Council (Vetenskapsrådet), the Swedish National Space Board and the research environment grant "Gravitational Radiation and Electromagnetic Astrophysical Transients (GREAT)." A. A.M. is funded by the Large Synoptic Survey Telescope Corporation, the Brinson Foundation, and the Moore Foundation in support of the LSSTC Data Science Fellowship Program; he also receives support as a CIERA Fellow by the CIERA Postdoctoral Fellowship Program (Center for Interdisciplinary Exploration and Research in Astrophysics, Northwestern University). M.R. has received funding from the European Research Council (ERC) under the European Union's Horizon 2020 research and innovation programme (grant agreement No. 759194 -USNAC). A.Y.Q.H. is supported by a National Science Foundation Graduate Research Fellowship under grant No. DGE1144469. M.L.G. acknowledges support from the DIRAC Institute in the Department of Astronomy at the University of Washington. The DIRAC Institute is supported through generous gifts from the Charles and Lisa Simonyi Fund for Arts and Sciences, and the Washington Research Foundation. These results made use of the Discovery Channel Telescope at Lowell Observatory. Lowell is a private, non-profit institution dedicated to astrophysical research and public appreciation of astronomy and operates the DCT in partnership with Boston University, the University of Maryland, the University of Toledo, Northern Arizona University and Yale University. The upgrade of the DeVeny optical spectrograph has been funded by a generous grant from John and Ginger Giovale and by a grant from the Mt. Cuba Astronomical Foundation. MMT Observatory access was supported by Northwestern University and the Center for Interdisciplinary Exploration and Research in Astrophysics (CIERA).

This work is based on observations obtained with the Samuel Oschin Telescope 48 inch and the 60 inch Telescope at the Palomar Observatory as part of the Zwicky Transient Facility project. ZTF is supported by the National Science Foundation 
under grant No. AST-1440341 and a collaboration including Caltech, IPAC, the Weizmann Institute for Science, the Oskar Klein Center at Stockholm University, the University of Maryland, the University of Washington, Deutsches Elektronen-Synchrotron and Humboldt University, Los Alamos National Laboratories, the TANGO Consortium of Taiwan, the University of Wisconsin at Milwaukee, and Lawrence Berkeley National Laboratories. Operations are conducted by COO, IPAC, and UW. SED Machine is based upon work supported by the National Science Foundation under grant No. 1106171.

Note added in proof. Following the acceptance of this paper, Fremling et al. 2019 demonstrated that the redshift of SNe Ia could be measured with SNID with a typical uncertainty of $\sim 0.004$. We have therefore updated the redshifts presented in Table 3, to reflect the Fremling et al. 2019 study, as we initially reported redshifts to two decimal places in cases where the redshift was determined via SNID, and now we report those redshifts to three decimal places. We have not, however, adopted these changes in the following analysis as the redshift differences result in negligible changes to the final results.

\section{ORCID iDs}

Yuhan Yao (10) https://orcid.org/0000-0001-6747-8509 Adam A. Miller (1) https://orcid.org/0000-0001-9515-478X S. R. Kulkarni (10 https://orcid.org/0000-0001-5390-8563 Mattia Bulla (iD https://orcid.org/0000-0002-8255-5127 Frank J. Masci (1D https://orcid.org/0000-0002-8532-9395 Daniel A. Goldstein (iD https://orcid.org/0000-0003-3461-8661 Ariel Goobar (ib https://orcid.org/0000-0002-4163-4996 Peter Nugent (iD https://orcid.org/0000-0002-3389-0586 Nadia Blagorodnova (D) https://orcid.org/0000-0003-0901-1606 James D. Neill (i) https://orcid.org/0000-0002-0466-1119 Mickael Rigault (i) https://orcid.org/0000-0002-8121-2560 Jesper Sollerman (ib https://orcid.org/0000-0003-1546-6615 Eric C. Bellm (iD https://orcid.org/0000-0001-8018-5348 S. Bradley Cenko (i) https://orcid.org/0000-0003-1673-970X Kishalay De (1) https://orcid.org/0000-0002-8989-0542 Ulrich Feindt (ib https://orcid.org/0000-0002-9435-2167 C. Fremling (i) https://orcid.org/0000-0002-4223-103X Pradip Gatkine (1) https://orcid.org/0000-0002-1955-2230 Matthew J. Graham (i) https://orcid.org/0000-0002-3168-0139 Melissa L. Graham (ib https://orcid.org/0000-0002-9154-3136 Anna Y. Q. Ho (1D https://orcid.org/0000-0002-9017-3567 T. Hung (D) https://orcid.org/0000-0002-9878-7889 Mansi M. Kasliwal (iD https://orcid.org/0000-0002-5619-4938 Thomas Kupfer (i) https://orcid.org/0000-0002-6540-1484 Russ R. Laher (iD https://orcid.org/0000-0003-2451-5482 Daniel A. Perley (ib https://orcid.org/0000-0001-8472-1996 Ben Rusholme (i) https://orcid.org/0000-0001-7648-4142 David L. Shupe (iD https://orcid.org/0000-0003-4401-0430 Maayane T. Soumagnac (iD https://orcid.org/0000-00016753-1488

K. Taggart (i) https://orcid.org/0000-0002-5748-4558

Lin Yan (iD https://orcid.org/0000-0003-1710-9339

\section{References}

Aldering, G., Antilogus, P., Bailey, S., et al. 2006, ApJ, 650, 510 Amanullah, R., Lidman, C., Rubin, D., et al. 2010, ApJ, 716, 712 Barbary, K., Barclay, T., Biswas, R., et al. 2016, SNCosmo: Python Library for Supernova Cosmology, Astrophysics Source Code Library, ascl:1611.017 Bellm, E. C. 2016, PASP, 128, 084501
Bellm, E. C., Kulkarni, S. R., Barlow, T., et al. 2019a, PASP, 131, 068003 Bellm, E. C., Kulkarni, S. R., Graham, M. J., et al. 2019b, PASP, 131, 018002 Betoule, M., Kessler, R., Guy, J., et al. 2014, A\&A, 568, A22

Bida, T. A., Dunham, E. W., Massey, P., \& Roe, H. G. 2014, Proc. SPIE, 9147, $91472 \mathrm{~N}$

Blagorodnova, N., Neill, J. D., Walters, R., et al. 2018, PASP, 130, 035003 Blondin, S., Matheson, T., Kirshner, R. P., et al. 2012, AJ, 143, 126 Blondin, S., \& Tonry, J. L. 2007, ApJ, 666, 1024

Bloom, J. S., Kasen, D., Shen, K. J., et al. 2012, ApJL, 744, L17

Cao, Y., Kulkarni, S. R., Howell, D. A., et al. 2015, Natur, 521, 328 Cardelli, J. A., Clayton, G. C., \& Mathis, J. S. 1989, ApJ, 345, 245

Cenko, S. B., Fox, D. B., Moon, D.-S., et al. 2006, PASP, 118, 1396 Contreras, C., Hamuy, M., Phillips, M. M., et al. 2010, AJ, 139, 519 De, K., Kasliwal, M. M., Polin, A., et al. 2019, ApJL, 873, L18 Dekany, R., Smith, R. M., Belicki, J., et al. 2016, Proc. SPIE, 9908, 99085M Dessart, L., Blondin, S., Hillier, D. J., \& Khokhlov, A. 2014, MNRAS, 441,532

Dilday, B., Howell, D. A., Cenko, S. B., et al. 2012, Sci, 337, 942

Dimitriadis, G., Foley, R. J., Rest, A., et al. 2019, ApJL, 870, L1

Fausnaugh, M. M., Vallely, P. J., Kochanek, C. S., et al. 2019, arXiv:1904. 02171

Filippenko, A. V., Li, W. D., Treffers, R. R., \& Modjaz, M. 2001, in ASP Conf. Ser. 246, IAU Coll. 183: Small Telescope Astronomy on Global Scales, ed. B. Paczynski, W.-P. Chen, \& C. Lemme (San Francisco, CA: ASP), 121

Filippenko, A. V., Richmond, M. W., Branch, D., et al. 1992a, AJ, 104, 1543 Filippenko, A. V., Richmond, M. W., Matheson, T., et al. 1992b, ApJL, 384, L15

Firth, R. E., Sullivan, M., Gal-Yam, A., et al. 2015, MNRAS, 446, 3895

Foley, R. J., Challis, P. J., Chornock, R., et al. 2013, ApJ, 767, 57

Foley, R. J., Matheson, T., Blondin, S., et al. 2009, AJ, 137, 3731

Foley, R. J., Scolnic, D., Rest, A., et al. 2018, MNRAS, 475, 193

Foreman-Mackey, D., Hogg, D. W., Lang, D., \& Goodman, J. 2013, PASP, 125,306

Fremling, U. C., Miller, A. A., Sharma, Y., et al. 2019, arXiv:1910.12973

Frieman, J. A., Bassett, B., Becker, A., et al. 2008, AJ, 135, 338

Ganeshalingam, M., Li, W., Filippenko, A. V., et al. 2010, ApJS, 190, 418

Ganeshalingam, M., Li, W., Filippenko, A. V., et al. 2012, ApJ, 751, 142

Gomez, S., Blanchard, P., Nicholl, M., Hosseinzadeh, G., \& Berger, E. 2018, ATel, 12052, 1

Goobar, A., Johansson, J., Amanullah, R., et al. 2014, ApJL, 784, L12

Goobar, A., Kromer, M., Siverd, R., et al. 2015, ApJ, 799, 106

Goodman, J., \& Weare, J. 2010, Communications in Applied Mathematics and Computational Science, 5, 65

Graham, M. J., Kulkarni, S. R., Bellm, E. C., et al. 2019, PASP, 131, 078001 Guy, J., Astier, P., Baumont, S., et al. 2007, A\&A, 466, 11

Hamuy, M., Folatelli, G., Morrell, N. I., et al. 2006, PASP, 118, 2

Hamuy, M., Phillips, M. M., Suntzeff, N. B., et al. 2003, Natur, 424, 651

Hayden, B. T., Garnavich, P. M., Kasen, D., et al. 2010a, ApJ, 722, 1691

Hayden, B. T., Garnavich, P. M., Kessler, R., et al. 2010b, ApJ, 712, 350

Hicken, M., Challis, P., Jha, S., et al. 2009, ApJ, 700, 331

Hicken, M., Challis, P., Kirshner, R. P., et al. 2012, ApJS, 200, 12

Hicken, M., Garnavich, P. M., Prieto, J. L., et al. 2007, ApJL, 669, L17

Hogg, D. W., Bovy, J., \& Lang, D. 2010, arXiv:1008.4686

Hosseinzadeh, G., Sand, D. J., Valenti, S., et al. 2017, ApJL, 845, L11

Howell, D. A., Sullivan, M., Nugent, P. E., et al. 2006, Natur, 443, 308

Jiang, J.-A., Doi, M., Maeda, K., et al. 2017, Natur, 550, 80

Kasen, D. 2010, ApJ, 708, 1025

Kasliwal, M. M., Cannella, C., Bagdasaryan, A., et al. 2019, PASP, 131, 038003

Krisciunas, K., Contreras, C., Burns, C. R., et al. 2017, AJ, 154, 211

Kromer, M., Fremling, C., Pakmor, R., et al. 2016, MNRAS, 459, 4428

Li, W., Filippenko, A. V., Chornock, R., \& Jha, S. 2003, PASP, 115, 844

Li, W., Filippenko, A. V., Treffers, R. R., et al. 2001, ApJ, 546, 734

Li, W., Wang, X., Vinko, J., et al. 2019, ApJ, 870, 12

Maeda, K., Jiang, J.-a., Shigeyama, T., \& Doi, M. 2018, ApJ, 861, 78

Maeda, K., \& Terada, Y. 2016, IJMPD, 25, 1630024

Malmquist, K. G. 1922, MeLuF, 100, 1

Maoz, D., Mannucci, F., \& Nelemans, G. 2014, ARA\&A, 52, 107

Marion, G. H., Brown, P. J., Vinkó, J., et al. 2016, ApJ, 820, 92

Masci, F. J., Laher, R. R., Rusholme, B., et al. 2019, PASP, 131, 018003

Miller, A. A., Cao, Y., Piro, A. L., et al. 2018, ApJ, 852, 100

Miller, A. A., Kasliwal, M. M., Cao, Y., et al. 2017, ApJ, 848, 59

Noebauer, U. M., Kromer, M., Taubenberger, S., et al. 2017, MNRAS, 472, 2787

Nugent, P. E., Sullivan, M., Cenko, S. B., et al. 2011, Natur, 480, 344 
Oke, J. B., Cohen, J. G., Carr, M., et al. 1995, PASP, 107, 375

Oke, J. B., \& Gunn, J. E. 1982, PASP, 94, 586

Ostman, L., Nordin, J., Goobar, A., et al. 2011, A\&A, 526, A28

Papadogiannakis, S., Goobar, A., Amanullah, R., et al. 2019, MNRAS, 483, 5045

Parrent, J., Friesen, B., \& Parthasarathy, M. 2014, Ap\&SS, 351, 1

Patterson, M. T., Bellm, E. C., Rusholme, B., et al. 2019, PASP, 131, 018001 Phillips, M. M. 1993, ApJL, 413, L105

Phillips, M. M., Contreras, C., Hsiao, E. Y., et al. 2019, PASP, 131, 014001

Phillips, M. M., Phillips, A. C., Heathcote, S. R., et al. 1987, PASP, 99, 592

Piro, A. L., \& Morozova, V. S. 2016, ApJ, 826, 96

Piro, A. L., \& Nakar, E. 2014, ApJ, 784, 85

Polin, A., Nugent, P., \& Kasen, D. 2019, ApJ, 873, 84

Ricker, G. R., Winn, J. N., Vanderspek, R., et al. 2015, JATIS, 1, 014003

Riess, A. G., Macri, L. M., Hoffmann, S. L., et al. 2016, ApJ, 826, 56

Rigault, M., Neill, J. D., Blagorodnova, N., et al. 2019, A\&A, 627, A115

Sako, M., Bassett, B., Becker, A. C., et al. 2018, PASP, 130, 064002

Scalzo, R. A., Aldering, G., Antilogus, P., et al. 2010, ApJ, 713, 1073

Schlafly, E. F., \& Finkbeiner, D. P. 2011, ApJ, 737, 103
Schlegel, D. J., Finkbeiner, D. P., \& Davis, M. 1998, ApJ, 500, 525

Schwarz, U. J. 1978, A\&A, 65, 345

Shappee, B. J., Holoien, T. W. S., Drout, M. R., et al. 2019, ApJ, 870, 13

Sharma, S. 2017, ARA\&A, 55, 213

Shen, K. J. 2015, ApJL, 805, L6

Silverman, J. M., Foley, R. J., Filippenko, A. V., et al. 2012, MNRAS, 425, 1789

Silverman, J. M., Nugent, P. E., Gal-Yam, A., et al. 2013, ApJS, 207, 3

Stetson, P. B. 1987, PASP, 99, 191

Stritzinger, M. D., Phillips, M. M., Boldt, L. N., et al. 2011, AJ, 142, 156

Taubenberger, S. 2017, in The Extremes of Thermonuclear Supernovae, ed. A. W. Alsabti \& P. Murdin (Cham: Springer International), 317

Tutukov, A., \& Yungelson, L. 1996, MNRAS, 280, 1035

Whelan, J., \& Iben, I., Jr. 1973, ApJ, 186, 1007

White, C. J., Kasliwal, M. M., Nugent, P. E., et al. 2015, ApJ, 799, 52

Woosley, S. E., \& Weaver, T. A. 1994, ApJ, 423, 371

Yamanaka, M., Kawabata, K. S., Kinugasa, K., et al. 2009, ApJL, 707, L118 Yaron, O., \& Gal-Yam, A. 2012, PASP, 124, 668

Zackay, B., Ofek, E. O., \& Gal-Yam, A. 2016, ApJ, 830, 27 